\title{
The laboratory analysis of $\mathrm{Bi} I I$ and its application to the $\mathrm{Bi}$-rich HgMn star HR 7775
}

\author{
L. Dolk, U. Litzén, and G. M. Wahlgren
}

Atomic Astrophysics, Department of Astronomy, Lund University, Box 43, 22100 Lund, Sweden

Received 20 February 2002 / Accepted 11 April 2002

\begin{abstract}
The bismuth spectrum emitted from a hollow cathode discharge has been recorded with a Fourier Transform Spectrometer (FTS). Accurate wavelengths have been determined for 104 Bi II lines, and several new energy levels have been found, while the accuracy of previously known Bi II level energies have been improved. The hyperfine structure of all observed Bi II lines has been analyzed, yielding hyperfine constants $A$ and $B$ for 56 Bi II levels. With the aid of the laboratory measurements the optical region spectrum of the HgMn star HR 7775 has been studied for all observable Bi II lines. The wavelengths and hfs constants established from the laboratory work have been combined with theoretical $g f$ values to identify spectral lines and make an abundance estimation of bismuth. It has been established that bismuth is present in HR 7775 at an enhancement level of approximately 5 orders of magnitude relative to the meteoritic abundance, consistent with previous observations in the ultraviolet region of this star. Astrophysical $g f$ values are presented for a number of Bi II lines.
\end{abstract}

Key words. atomic data - stars: chemically peculiar - stars: individual: HR 7775

\section{Introduction}

The chemically peculiar $(\mathrm{CP})$ stars of the upper main sequence are recognized by their anomalous abundances for a variety of elements. In a subgroup of $\mathrm{CP}$ stars, the $\mathrm{HgMn}$ stars, the abundance enhancements of the very heavy elements, $\mathrm{Pt}, \mathrm{Au}, \mathrm{Hg}$ are particularly noticeable, with line depths indicating enhancements of up to $10^{6}$ times the solar value. The two heaviest stable elements, $\mathrm{Pb}$ and $\mathrm{Bi}$, have not, however, been observed at a similar enhancement level in HgMn stars, with the exception of bismuth in the HgMn star HR 7775.

The presence of bismuth features in stellar spectra has been reported in a few different type of stars. Guthrie (1972) reported the identification of one Bi I feature in the Ap star 73 Dra. Several strong Bi II features were observed in the ultraviolet region of the HgMn star HR 7775 (Jacobs \& Dworetsky 1982), and subsequent synthetic calculations indicated an overabundance of bismuth of approximately $10^{6}$ times compared to the solar system composition. Jacobs \& Dworetsky noted that out of a sample of $13 \mathrm{HgMn}$ stars, for which IUE data were analysed, only HR 7775 showed evidence of strong features at the laboratory position of strong BiII features. The discovery of the strong ultraviolet Bi II lines in HR 7775 by Jacobs \& Dworetsky prompted the tentative identification of an optical line at $\lambda 4259$ as Bi II (Guthrie 1984). Two lines

Send offprint requests to: L. Dolk, e-mail: linus.dolk@astro.lu.se of Bi II were identified on the basis of wavelength coincidence statistics (WCS) from the IUE spectrum of the magnetic Ap star HR 465 (Cowley 1987), and this tentative identification was later substantiated by Fuhrmann (1989). Fuhrmann also reported the possible existence of Bi II features in the Ap star $\alpha^{2} \mathrm{CVn}$.

More recently, selected Bi II lines have been studied in the ultraviolet spectrum of the $\mathrm{HgMn}$ star $\chi$ Lupi (Wahlgren et al. 1994; Leckrone et al. 1998, 1999), with the conclusion that the bismuth abundance is only marginally enhanced in this star. The investigations were made utilizing high-resolution data obtained with the Goddard High Resolution Spectrograph (GHRS) on the Hubble Space Telescope (HST). A recent paper, also utilizing the HST data, considers the bismuth abundance in the two $\mathrm{HgMn}$ stars $\chi$ Lupi and HR 7775 (Wahlgren et al. 2001). An approximate abundance enhancement of 5 dex for bismuth is noted for HR 7775 , while only a moderate abundance enhancement of 0.9 dex is noted for $\chi$ Lupi. Thus, the absence of bismuth features in the IUE spectrum of all $\mathrm{HgMn}$ stars (except HR 7775) observed by Jacobs \& Dworetsky, does not preclude the possibility of a moderate bismuth enhancement in these stars.

It was noted that several unidentified features in the optical region spectrum of HR 7775 coincide with the laboratory positions of Bi II features (Wahlgren et al. 2000). Since several of these features had not been studied for hfs, this served as an incentive to investigate the Bi II spectrum in the laboratory. 
The term system of Bi II presented in Atomic Energy Levels (AEL) (Moore 1958) is based on the laboratory investigation of the Bi II spectrum made by Crawford \& McLay (1934) as revised by Murakawa \& Suwa (1947). In the paper by Crawford \& McLay the spark spectra of Bi II and $\mathrm{Bi}$ III were studied and the wavelengths of all observable lines between $1000 \AA$ and $1 \mu \mathrm{m}$ were measured. It was noted that most of the observed lines had unresolved hyperfine structure (hfs), and hfs intervals were presented for several energy levels.

No general investigation of the Bi II spectrum has been made since the analysis by Crawford \& McLay, although accurate wavelength measurements of all observable lines in the 1058-3117 $\AA$ region were presented by Wahlgren et al. (2001). These measurements were made at the laboratory resolving power $(R=\lambda / \Delta \lambda=150000)$ and wavelengths were reported for all the observed $\mathrm{Bi}$ II features, including the hfs components of lines with large enough hfs splittings. There have been several investigations regarding the structure of individual Bi II lines. The hfs of the lowest lying odd and even Bi II configurations have been studied by Cole (1964), Arcimowicz \& Dembczynski (1979), Augustyniak \& Werel (1984) and Bouazza \& Bauche (1988), while the hfs of selected higher excitation energy levels have been presented by Eisele et al. (1968), Stachowska (1987), Stachowska et al. (1987) and Grabowski et al. (1996).

In this paper a thorough laboratory analysis of the Bi II spectrum has been made with a high resolution Fourier Transform Spectrometer (FTS). The specific aim was to improve existing absolute wavelengths and energy levels of Bi II and to analyse the hfs of a large number of Bi II lines for the benefit of astrophysical studies of bismuth in stellar spectra. The laboratory data have been utilized to study several optical region Bi II lines in the spectrum of the HgMn star HR 7775.

\section{Experiment}

The bismuth spectrum was emitted from a hollow cathode discharge, with the cathode consisting of a water cooled $50 \mathrm{~mm}$ long bismuth tube with the inner diameter $5 \mathrm{~mm}$. The discharge was run at $450 \mathrm{~mA}$ with neon at 1.5 Torr or argon at 0.5 Torr as carrier gas. The spectra were recorded with a Chelsea Instruments FT500 Fourier transform spectrometer in three overlapping spectral regions, together covering the range 2000-7000 $\AA$. The wavenumber scale was calibrated by means of Ar II lines (Norlén 1973).

Spectral lines from high Bi II levels were enhanced in the Bi:Ne discharge through overpopulation of the high levels by the charge and energy transfer reaction

$\mathrm{Bi}+\mathrm{Ne}^{+} \rightarrow \mathrm{Bi}^{+*}+\mathrm{Ne}+\Delta E$,

where the increase of the population has a maximum at $\Delta E$ in the range $0.5-1 \mathrm{eV}$ (Johansson \& Litzén 1978). This would give an enhancement of lines from Bi II levels in the region $105000-115000 \mathrm{~cm}^{-1}$ above the Ground state, which is indeed observed. Unexpectedly strong lines are also observed from levels around $125000-127000 \mathrm{~cm}^{-1}$, which may be caused by collisions between $\mathrm{Ne}^{+}$ions and bismuth atoms in the metastable $6 \mathrm{p}^{3}{ }^{2} \mathrm{D}$ levels 11419 and $15437 \mathrm{~cm}^{-1}$ above the Ground state. An example of the enhancement is shown in Fig. 1.

\section{Analysis of the hyperfine structure}

The hfs in all Bi II transitions was analyzed by means of a program in the computer code package IDL (Interactive Data Language, Research Systems, Inc., Boulder), where parameters of a predicted function were fitted to the observed feature. The predicted function, a sum of Gaussians, was based on the following assumptions (as given in Karlsson \& Litzén 2001):

1. Each hfs component has a Doppler profile.

2. The hyperfine level splitting depends on the magnetic dipole and the electric quadrupole interaction according to

$$
\Delta E=\frac{1}{2} A C+\frac{B}{4} \frac{\frac{3}{2} C(C+1)-2 I(I+1) J(J+1)}{I(2 I-1) J(2 J-1)}
$$

where $C=F(F+1)-I(I+1)-J(J+1)$.

3 . The intensities of the individual components are distributed according to the corresponding line strength relations in a multiplet at pure $L S$ coupling.

The free parameters in the analysis were the centre-ofgravity wavenumber of the hfs-pattern, the intensity of the strongest component, the Doppler width of the components and the hfs constants $A$ and $B$ for the upper and the lower level.

In the analysis transitions where one of the levels had $J=0$ and the other level $J=1$ were first studied. This means that the feature will depend on only one $A$ and one $B$ parameter and only consist of three well resolved components. The fitting routine is then rather insensitive to the starting values of the parameters. Then successively more complicated structures were analyzed, where the $A$ and $B$ from a previous fit could be fixed or used as starting values for one of the involved levels. In the cases where the $A$ and $B$ parameters of a level were fixed for the analysis of a line, a second run was made allowing the values to be changed. This was done in order to reduce a propagation of errors in the $A$ and $B$ values.

\section{The Bill spectral lines and energy levels}

All the Bi II spectral lines observed and analyzed in the present work are shown in Table 1 . The wavenumbers in column three represent the centres-of-gravity of the hfs patterns, derived as described in the previous section. The corresponding wavelengths are shown in column two, where the air wavelengths above $2000 \AA$ were derived by means of Edlén's dispersion formula (Edlén 1966). The uncertainty of the wavenumbers is estimated as varying 


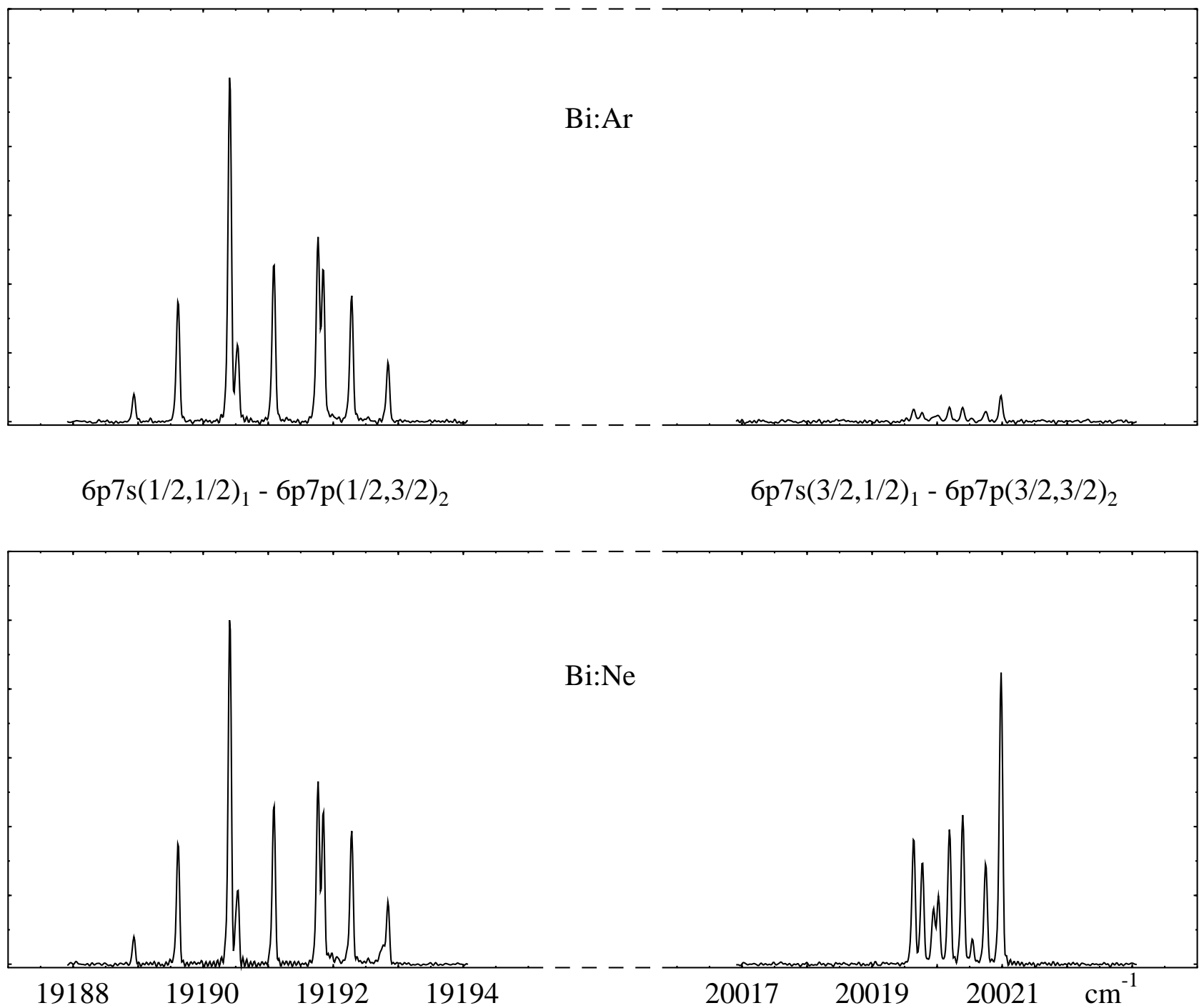

Fig. 1. An example of the enhancement of a Bi II line in the Bi:Ne discharge through overpopulation of a high level caused by collisions between $\mathrm{Ne}^{+}$ions and bismuth atoms.

from $0.003 \mathrm{~cm}^{-1}$ for strong lines with well resolved hfs patterns to $0.05 \mathrm{~cm}^{-1}$ for weak lines with complex hfs. This corresponds to $0.001-0.02 \AA$ at $6000 \AA$ and $0.0001-0.002 \AA$ at $2000 \AA$. The intensity noted in the first column represents the signal-to-noise ratio (SNR) of the strongest peak of the observed hfs pattern, and is thus strongly affected by the charge and energy transfer reactions in the light source. The fourth column shows the difference between the observed wavenumber of a line and the wavenumber derived from the improved energy level values (see below). This difference is only shown for lines where more than one line was used for establishing the upper energy level. The last column of the table contains the designations of the combining levels as discussed below.

Besides the lines observed in the present work the table also contains three lines below $2000 \AA$, viz. the $\lambda 1902$ line from Wahlgren et al. (1994) and $\lambda 1436$ and $\lambda 1791$ from Wahlgren et al. (2001). These lines were used for providing accurate connections between the Bi II Ground state and the excited configurations.
The energy level values derived from the lines of Table 1 are shown in Tables 2 and 3, and an overview of the energy level system can be seen in Fig. 2. The level values were optimized by means of the computer code ELCALC (Radziemski et al. 1972). The uncertainty of the relative energy level values of the excited configurations is estimated as varying from $0.002 \mathrm{~cm}^{-1}$ for levels connected by several lines to $0.05 \mathrm{~cm}^{-1}$ for levels established by only one, weak line. The uncertainty of the values of the absolute energy levels relative to the ground term depends on the accuracy of the line at $1436 \AA$, stated in Wahlgren et al. (2001) as $0.0010 \AA$ or $0.05 \mathrm{~cm}^{-1}$.

Tables 2 and 3 also contain the hyperfine constants $A$ and $B$ derived from the analysis of the hfs patterns as described in a previous section. For levels involved in more than one transition the $A$ and $B$ values are averaged from the different transitions, weighted according to the SNR of the observed feature. The uncertainties shown in the tables represent estimated fitting errors of the derived hfs constants. These errors were determined 
Table 1. Observed Bi II lines.

\begin{tabular}{|c|c|c|c|c|c|c|}
\hline $\operatorname{Int}^{a}$ & $\lambda^{b}(\AA)$ & $\sigma\left(\mathrm{cm}^{-1}\right)$ & $o-c$ & \multicolumn{3}{|c|}{ Combination } \\
\hline 24 & 6809.1955 & 14681.971 & 0.000 & $6 \mathrm{p} 7 \mathrm{~s}(1 / 2,1 / 2)_{1}$ & - & $6 \mathrm{p} 7 \mathrm{p}(1 / 2,1 / 2)_{1}$ \\
\hline 26 & 6600.3388 & 15146.554 & -0.001 & $6 \mathrm{p} 7 \mathrm{~s}(1 / 2,1 / 2)_{0}$ & - & $6 \mathrm{p} 7 \mathrm{p}(1 / 2,1 / 2)_{1}$ \\
\hline 5 & 6059.1102 & 16499.505 & & $6 \mathrm{p} 7 \mathrm{~s}(3 / 2,1 / 2)_{2}$ & - & $6 \mathrm{p} 7 \mathrm{p}(3 / 2,1 / 2)_{1}$ \\
\hline 58 & 5719.1384 & 17480.302 & 0.000 & $6 \mathrm{p} 7 \mathrm{~s}(1 / 2,1 / 2)_{1}$ & - & $6 \mathrm{p} 7 \mathrm{p}(1 / 2,1 / 2)_{0}$ \\
\hline 6 & 5655.1659 & 17678.041 & -0.003 & $6 \mathrm{p} 7 \mathrm{~s}(3 / 2,1 / 2)_{2}$ & - & $6 \mathrm{p} 5 \mathrm{f}(1 / 2,5 / 2)_{2}$ \\
\hline 3 & 5501.2997 & 18172.474 & 0.002 & $6 \mathrm{p} 7 \mathrm{~d}(1 / 2,3 / 2)_{1}$ & - & $6 \mathrm{p} 5 \mathrm{f}(3 / 2,5 / 2)_{2}$ \\
\hline 6 & 5490.3705 & 18208.648 & 0.000 & $6 \mathrm{~s} 6 \mathrm{p}^{3}{ }^{3} \mathrm{D}_{1}$ & - & $6 \mathrm{p} 7 \mathrm{p}(3 / 2,3 / 2)_{0}$ \\
\hline 4 & 5397.8894 & 18520.610 & 0.001 & $6 \mathrm{p} 7 \mathrm{~s}(3 / 2,1 / 2)_{1}$ & - & $6 \mathrm{p} 7 \mathrm{p}(3 / 2,3 / 2)_{1}$ \\
\hline 8 & 5361.9475 & 18644.755 & -0.001 & $6 \mathrm{p} 6 \mathrm{~d}(3 / 2,5 / 2)_{3}$ & - & $6 \mathrm{p} 5 \mathrm{f}(3 / 2,5 / 2)_{4}$ \\
\hline 31 & 5270.5120 & 18968.210 & 0.000 & $6 \mathrm{p} 7 \mathrm{~s}(1 / 2,1 / 2)_{1}$ & - & $6 \mathrm{p} 7 \mathrm{p}(1 / 2,3 / 2)_{1}$ \\
\hline 4 & 5245.8158 & 19057.507 & -0.003 & $6 \mathrm{p} 7 \mathrm{~d}(1 / 2,3 / 2)_{1}$ & - & $6 \mathrm{p} 5 \mathrm{f}(3 / 2,7 / 2)_{2}$ \\
\hline 191 & 5209.3246 & 19191.003 & 0.000 & $6 \mathrm{p} 7 \mathrm{~s}(1 / 2,1 / 2)_{1}$ & - & $6 \mathrm{p} 7 \mathrm{p}(1 / 2,3 / 2)_{2}$ \\
\hline 2 & 5201.5800 & 19219.576 & -0.003 & $6 \mathrm{p} 7 \mathrm{p}(1 / 2,3 / 2)_{2}$ & - & $6 \mathrm{p} 6 \mathrm{~d}(3 / 2,5 / 2)_{3}$ \\
\hline 2 & 5201.5232 & 19219.786 & 0.000 & $6 \mathrm{p} 7 \mathrm{~s}(3 / 2,1 / 2)_{1}$ & - & $6 \mathrm{p} 8 \mathrm{p}(1 / 2,3 / 2)_{1}$ \\
\hline 121 & 5144.4921 & 19432.851 & 0.057 & $6 \mathrm{p} 7 \mathrm{~s}(1 / 2,1 / 2)_{0}$ & - & $6 \mathrm{p} 7 \mathrm{p}(1 / 2,3 / 2)_{1}$ \\
\hline 57 & 5124.3561 & 19509.211 & 0.001 & $6 \mathrm{p} 7 \mathrm{~s}(3 / 2,1 / 2)_{2}$ & - & $6 \mathrm{p} 7 \mathrm{p}(3 / 2,3 / 2)_{3}$ \\
\hline 10 & 5091.5684 & 19634.841 & 0.000 & $6 \mathrm{p} 7 \mathrm{~s}(3 / 2,1 / 2)_{2}$ & - & $6 \mathrm{p} 7 \mathrm{p}(3 / 2,3 / 2)_{1}$ \\
\hline 157 & 4993.5338 & 20020.313 & 0.001 & $6 \mathrm{p} 7 \mathrm{~s}(3 / 2,1 / 2)_{1}$ & - & $6 \mathrm{p} 7 \mathrm{p}(3 / 2,3 / 2)_{2}$ \\
\hline 8 & 4969.5134 & 20117.081 & 0.005 & $6 \mathrm{p} 7 \mathrm{~s}(3 / 2,1 / 2)_{2}$ & - & $6 \mathrm{p} 8 \mathrm{p}(1 / 2,3 / 2)_{2}$ \\
\hline 5 & 4876.6967 & 20499.958 & 0.001 & $6 \mathrm{p} 5 \mathrm{f}(1 / 2,5 / 2)_{2}$ & - & $6 \mathrm{p} 7 \mathrm{~d}(3 / 2,3 / 2)_{3}$ \\
\hline 7 & 4749.7270 & 21047.955 & -0.001 & $6 \mathrm{p} 7 \mathrm{p}(1 / 2,1 / 2)_{0}$ & - & $6 \mathrm{p} 7 \mathrm{~d}(1 / 2,3 / 2)_{1}$ \\
\hline 74 & 4730.2672 & 21134.543 & -0.001 & $6 \mathrm{p} 7 \mathrm{~s}(3 / 2,1 / 2)_{2}$ & - & $6 \mathrm{p} 7 \mathrm{p}(3 / 2,3 / 2)_{2}$ \\
\hline 72 & 4705.2854 & 21246.751 & & $6 \mathrm{p} 7 \mathrm{p}(1 / 2,1 / 2)_{1}$ & - & $6 \mathrm{p} 7 \mathrm{~d}(1 / 2,3 / 2)_{2}$ \\
\hline 7 & 4572.4659 & 21863.910 & -0.002 & $6 \mathrm{p} 7 \mathrm{p}(3 / 2,1 / 2)_{2}$ & - & $6 \mathrm{p} 7 \mathrm{~d}(3 / 2,3 / 2)_{3}$ \\
\hline 2 & 4493.9702 & 22245.798 & 0.004 & $6 \mathrm{p} 6 \mathrm{~d}(3 / 2,3 / 2)_{3}$ & - & $6 \mathrm{p} 8 \mathrm{f}(1 / 2,7 / 2)_{4}$ \\
\hline 5 & 4466.5746 & 22382.240 & -0.001 & $6 \mathrm{p} 6 \mathrm{~d}(3 / 2,3 / 2)_{3}$ & - & $6 \mathrm{p} 5 \mathrm{f}(3 / 2,5 / 2)_{3}$ \\
\hline 2 & 4436.6820 & 22533.040 & -0.005 & $6 \mathrm{p} 6 \mathrm{~d}(3 / 2,3 / 2)_{3}$ & - & $6 \mathrm{p} 5 \mathrm{f}(3 / 2,7 / 2)_{4}$ \\
\hline 188 & 4391.4358 & 22765.200 & 0.000 & $6 \mathrm{p} 7 \mathrm{~s}(3 / 2,1 / 2)_{1}$ & - & $6 \mathrm{p} 7 \mathrm{p}(3 / 2,3 / 2)_{0}$ \\
\hline 8 & 4340.4737 & 23032.485 & 0.004 & $6 \mathrm{p} 6 \mathrm{~d}(1 / 2,5 / 2)_{3}$ & - & $6 \mathrm{p} 5 \mathrm{f}(1 / 2,7 / 2)_{3}$ \\
\hline 3 & 4339.8307 & 23035.897 & -0.003 & $6 \mathrm{p} 6 \mathrm{~d}(1 / 2,5 / 2)_{2}$ & - & $6 \mathrm{p} 7 \mathrm{p}(3 / 2,1 / 2)_{2}$ \\
\hline 2 & 4336.4052 & 23054.094 & 0.016 & $6 \mathrm{p} 6 \mathrm{~d}(3 / 2,3 / 2)_{2}$ & - & $6 \mathrm{p} 5 \mathrm{f}(3 / 2,5 / 2)_{3}$ \\
\hline 64 & 4301.6974 & 23240.100 & 0.000 & $6 \mathrm{p} 6 \mathrm{~d}(1 / 2,5 / 2)_{2}$ & - & $6 \mathrm{p} 5 \mathrm{f}(1 / 2,7 / 2)_{3}$ \\
\hline 17 & 4272.0440 & 23401.413 & 0.000 & $6 \mathrm{p} 6 \mathrm{~d}(1 / 2,5 / 2)_{2}$ & - & $6 \mathrm{p} 5 \mathrm{f}(1 / 2,5 / 2)_{3}$ \\
\hline 124 & 4259.4126 & 23470.809 & & $6 \mathrm{p} 6 \mathrm{~d}(1 / 2,5 / 2)_{3}$ & - & $6 \mathrm{p} 5 \mathrm{f}(1 / 2,7 / 2)_{4}$ \\
\hline 50 & 4227.0843 & 23650.308 & 0.000 & $6 \mathrm{p} 6 \mathrm{~d}(3 / 2,3 / 2)_{3}$ & - & $6 \mathrm{p} 5 \mathrm{f}(3 / 2,5 / 2)_{4}$ \\
\hline 54 & 4204.7421 & 23775.973 & -0.001 & $6 \mathrm{p} 6 \mathrm{~d}(3 / 2,3 / 2)_{2}$ & - & $6 \mathrm{p} 5 \mathrm{f}(3 / 2,7 / 2)_{3}$ \\
\hline 6 & 4171.1345 & 23967.537 & 0.000 & $6 \mathrm{p} 6 \mathrm{~d}(3 / 2,3 / 2)_{2}$ & - & $6 \mathrm{p} 5 \mathrm{f}(3 / 2,5 / 2)_{2}$ \\
\hline 3 & 4097.2286 & 24399.855 & 0.000 & $6 \mathrm{p} 6 \mathrm{~d}(1 / 2,5 / 2)_{2}$ & - & $6 \mathrm{p} 5 \mathrm{f}(1 / 2,5 / 2)_{2}$ \\
\hline 99 & 4079.0719 & 24508.461 & 0.000 & $6 \mathrm{p} 6 \mathrm{~d}(1 / 2,3 / 2)_{1}$ & - & $6 \mathrm{p} 7 \mathrm{p}(3 / 2,1 / 2)_{2}$ \\
\hline 2 & 4022.5909 & 24852.576 & 0.001 & $6 \mathrm{p} 6 \mathrm{~d}(3 / 2,3 / 2)_{2}$ & - & $6 \mathrm{p} 5 \mathrm{f}(3 / 2,7 / 2)_{2}$ \\
\hline 6 & 4005.4745 & 24958.775 & 0.000 & $6 \mathrm{p} 8 \mathrm{~s}(1 / 2,1 / 2)_{1}$ & - & $6 \mathrm{p} 5 \mathrm{f}(3 / 2,5 / 2)_{2}$ \\
\hline 6 & 3905.2689 & 25599.180 & 0.000 & $6 \mathrm{p} 8 \mathrm{~s}(1 / 2,1 / 2)_{0}$ & - & $6 \mathrm{p} 5 \mathrm{f}(3 / 2,5 / 2)_{1}$ \\
\hline 41 & 3874.2291 & 25804.272 & -0.002 & $6 \mathrm{p} 6 \mathrm{~d}(3 / 2,3 / 2)_{1}$ & - & $6 \mathrm{p} 5 \mathrm{f}(3 / 2,5 / 2)_{2}$ \\
\hline 55 & 3871.2853 & 25823.894 & 0.000 & $6 \mathrm{p} 7 \mathrm{p}(1 / 2,3 / 2)_{2}$ & - & $6 \mathrm{p} 9 \mathrm{~s}(1 / 2,1 / 2)_{1}$ \\
\hline 70 & 3864.0245 & 25872.418 & 0.002 & 6p6d $(1 / 2,3 / 2)_{1}$ & - & $6 \mathrm{p} 5 \mathrm{f}(1 / 2,5 / 2)_{2}$ \\
\hline 10 & 3845.8928 & 25994.392 & 0.001 & $6 \mathrm{p} 6 \mathrm{~d}(1 / 2,3 / 2)_{2}$ & - & $6 \mathrm{p} 7 \mathrm{p}(3 / 2,1 / 2)_{2}$ \\
\hline 34 & 3843.2278 & 26012.417 & 0.001 & $6 \mathrm{p} 7 \mathrm{p}(1 / 2,3 / 2)_{1}$ & - & $6 \mathrm{p} 9 \mathrm{~s}(1 / 2,1 / 2)_{0}$ \\
\hline 10 & 3838.1712 & 26046.686 & -0.001 & $6 \mathrm{p} 7 \mathrm{p}(1 / 2,3 / 2)_{1}$ & - & $6 \mathrm{p} 9 \mathrm{~s}(1 / 2,1 / 2)_{1}$ \\
\hline 35 & 3827.0261 & 26122.538 & 0.000 & $6 \mathrm{p} 6 \mathrm{~d}(3 / 2,5 / 2)_{2}$ & - & $6 \mathrm{p} 8 \mathrm{f}(1 / 2,5 / 2)_{3}$ \\
\hline 33 & 3815.9162 & 26198.591 & 0.000 & $6 \mathrm{p} 6 \mathrm{~d}(1 / 2,3 / 2)_{2}$ & - & $6 \mathrm{p} 5 \mathrm{f}(1 / 2,7 / 2)_{3}$ \\
\hline 15 & $3812.5219^{c}$ & 26221.915 & & $6 \mathrm{p} 6 \mathrm{~d}(1 / 2,5 / 2)_{4}$ & - & 6p5f $\left(1 / 2,7 / 29_{5}\right.$ \\
\hline 17 & 3811.1984 & 26231.021 & 0.000 & $6 \mathrm{p} 6 \mathrm{~d}(1 / 2,5 / 2)_{2}$ & - & $6 \mathrm{p} 7 \mathrm{p}(3 / 2,3 / 2)_{3}$ \\
\hline 203 & 3792.5636 & 26359.904 & 0.000 & $6 \mathrm{p} 6 \mathrm{~d}(1 / 2,3 / 2)_{2}$ & - & $6 \mathrm{p} 5 \mathrm{f}(1 / 2,5 / 2)_{3}$ \\
\hline 32 & 3790.4766 & 26374.417 & -0.001 & $6 \mathrm{p} 6 \mathrm{~d}(3 / 2,5 / 2)_{2}$ & - & $6 \mathrm{p} 5 \mathrm{f}(3 / 2,5 / 2)_{3}$ \\
\hline 22 & 3762.9021 & 26567.684 & 0.000 & $6 \mathrm{p} 6 \mathrm{~d}(3 / 2,3 / 2)_{1}$ & - & $6 \mathrm{p} 5 \mathrm{f}(3 / 2,5 / 2)_{1}$ \\
\hline 17 & 3745.7530 & 26689.315 & 0.003 & $6 \mathrm{p} 6 \mathrm{~d}(3 / 2,3 / 2)_{1}$ & - & $6 \mathrm{p} 5 \mathrm{f}(3 / 2,7 / 2)_{2}$ \\
\hline 32 & 3719.1568 & 26880.169 & 0.000 & $6 \mathrm{p} 6 \mathrm{~d}(3 / 2,3 / 2)_{0}$ & - & $6 \mathrm{p} 5 \mathrm{f}(3 / 2,5 / 2)_{1}$ \\
\hline 25 & 3689.4887 & 27096.314 & 0.000 & $6 \mathrm{p} 6 \mathrm{~d}(3 / 2,5 / 2)_{2}$ & - & $6 \mathrm{p} 5 \mathrm{f}(3 / 2,7 / 2)_{3}$ \\
\hline 8 & 3654.1507 & 27358.346 & 0.000 & $6 \mathrm{p} 6 \mathrm{~d}(1 / 2,3 / 2)_{2}$ & - & $6 \mathrm{p} 5 \mathrm{f}(1 / 2,5 / 2)_{2}$ \\
\hline 18 & 3630.7598 & 27534.595 & 0.000 & $6 \mathrm{p} 7 \mathrm{p}(1 / 2,1 / 2)_{0}$ & - & $6 \mathrm{p} 9 \mathrm{~s}(1 / 2,1 / 2)_{1}$ \\
\hline
\end{tabular}


Table 1. continued.

\begin{tabular}{|c|c|c|c|c|c|c|}
\hline $\operatorname{Int}^{a}$ & $\lambda^{b}(\AA)$ & $\sigma\left(\mathrm{cm}^{-1}\right)$ & $o-c$ & \multicolumn{3}{|c|}{ Combination } \\
\hline 4 & 3615.7701 & 27648.741 & 0.005 & $6 \mathrm{p} 6 \mathrm{~d}(1 / 2,5 / 2)_{3}$ & - & $6 \mathrm{p} 7 \mathrm{p}(3 / 2,3 / 2)_{2}$ \\
\hline 8 & 3523.0817 & 28376.128 & 0.000 & $6 \mathrm{p} 7 \mathrm{p}(1 / 2,3 / 2)_{1}$ & - & $6 \mathrm{p} 8 \mathrm{~d}(1 / 2,5 / 2)_{2}$ \\
\hline 23 & 3515.7776 & 28435.078 & & $6 \mathrm{p} 7 \mathrm{p}(1 / 2,3 / 2)_{2}$ & - & $6 \mathrm{p} 8 \mathrm{~d}(1 / 2,5 / 2)_{3}$ \\
\hline 47 & 3430.6054 & 29141.019 & -0.001 & $6 \mathrm{~s} \mathrm{p}^{3}{ }^{5} \mathrm{~S}_{2}$ & - & $6 \mathrm{p} 5 \mathrm{f}(1 / 2,7 / 2)_{3}$ \\
\hline 10 & 3425.2072 & 29186.945 & 0.002 & $6 \mathrm{~s} 6 \mathrm{p}^{3}{ }^{3} \mathrm{D}_{3}$ & - & $6 \mathrm{p} 8 \mathrm{f}(1 / 2,7 / 2)_{4}$ \\
\hline 5 & 3409.2689 & 29323.389 & -0.001 & $6 \mathrm{~s} 6 \mathrm{p}^{3}{ }^{3} \mathrm{D}_{3}$ & - & $6 \mathrm{p} 5 \mathrm{f}(3 / 2,5 / 2)_{3}$ \\
\hline 12 & 3408.6264 & 29328.916 & 0.000 & $6 \mathrm{p} 6 \mathrm{~d}(1 / 2,3 / 2)_{1}$ & - & $6 \mathrm{p} 7 \mathrm{p}(3 / 2,3 / 2)_{2}$ \\
\hline 51 & 3391.8248 & 29474.194 & 0.000 & $6 \mathrm{~s} 6 \mathrm{p}^{3}{ }^{3} \mathrm{D}_{3}$ & - & $6 \mathrm{p} 5 \mathrm{f}(3 / 2,7 / 2)_{4}$ \\
\hline 13 & 3327.3521 & 30045.285 & -0.001 & $6 s 6 p^{3}{ }^{3} D_{3}$ & - & $6 \mathrm{p} 5 \mathrm{f}(3 / 2,7 / 2)_{3}$ \\
\hline 12 & 3309.9520 & 30203.225 & -0.001 & $6 s 6 \mathrm{p}^{3}{ }^{3} \mathrm{D}_{2}$ & - & $6 \mathrm{p} 8 \mathrm{f}(1 / 2,5 / 2)_{3}$ \\
\hline 15 & 3299.5266 & 30298.654 & -0.001 & $6 \mathrm{p} 7 \mathrm{p}(1 / 2,1 / 2)_{1}$ & - & $6 \mathrm{p} 9 \mathrm{~s}(1 / 2,1 / 2)_{0}$ \\
\hline 17 & 3295.7984 & 30332.926 & 0.000 & $6 \mathrm{p} 7 \mathrm{p}(1 / 2,1 / 2)_{1}$ & - & $6 \mathrm{p} 9 \mathrm{~s}(1 / 2,1 / 2)_{1}$ \\
\hline 36 & 3282.5759 & 30455.106 & 0.000 & $6 \mathrm{~s}^{2} \mathrm{p}^{3}{ }^{3} \mathrm{D}_{2}$ & - & $6 \mathrm{p} 5 \mathrm{f}(3 / 2,5 / 2)_{3}$ \\
\hline 8 & 3186.9830 & 31368.568 & 0.003 & $6 \mathrm{~s} 6 \mathrm{p}^{3}{ }^{3} \mathrm{D}_{2}$ & - & $6 \mathrm{p} 5 \mathrm{f}(3 / 2,5 / 2)_{2}$ \\
\hline 25 & 3116.9054 & 32073.803 & -0.001 & $6 \mathrm{p} 6 \mathrm{~d}(1 / 2,3 / 2)_{1}$ & - & $6 \mathrm{p} 7 \mathrm{p}(3 / 2,3 / 2)_{0}$ \\
\hline 3 & 3111.2661 & 32131.936 & -0.005 & $6 \mathrm{~s} \mathrm{p}^{3}{ }^{5} \mathrm{~S}_{2}$ & - & $6 \mathrm{p} 7 \mathrm{p}(3 / 2,3 / 2)_{3}$ \\
\hline 5 & 3060.7375 & 32662.369 & 0.002 & $6 \mathrm{p} 7 \mathrm{p}(1 / 2,1 / 2)_{1}$ & - & $6 \mathrm{p} 8 \mathrm{~d}(1 / 2,5 / 2)_{2}$ \\
\hline 2 & 2968.3174 & 33679.285 & -0.020 & $6 \mathrm{p} 6 \mathrm{~d}(1 / 2,5 / 2)_{3}$ & - & $6 \mathrm{p} 6 \mathrm{f}(1 / 2,5 / 2)_{3}$ \\
\hline 25 & 2950.4167 & 33883.615 & & $6 \mathrm{p} 6 \mathrm{~d}(1 / 2,5 / 2)_{3}$ & - & 6p6f $(1 / 2,7 / 2)_{4}$ \\
\hline 12 & 2936.7450 & 34041.349 & 0.001 & 6p6d $(1 / 2,5 / 2)_{2}$ & - & 6p6f $(1 / 2,7 / 2)_{3}$ \\
\hline 31 & 2805.2213 & 35637.311 & -0.001 & $6 \mathrm{p} 6 \mathrm{~d}(1 / 2,3 / 2)_{1}$ & - & 6p6f $(1 / 2,5 / 2)_{2}$ \\
\hline 12 & 2803.4556 & 35659.756 & -0.001 & $6 \mathrm{p}^{2}{ }^{1} \mathrm{D}_{2}$ & - & $6 \mathrm{p} 7 \mathrm{~s}(1 / 2,1 / 2)_{1}$ \\
\hline 2 & 2745.4201 & 36413.528 & -0.002 & $6 \mathrm{p} 7 \mathrm{~s}(3 / 2,1 / 2)_{1}$ & - & $6 \mathrm{p} 5 \mathrm{f}(3 / 2,5 / 2)_{2}$ \\
\hline 100 & 2713.2377 & 36845.415 & 0.000 & $6 \mathrm{p} 6 \mathrm{~d}(1 / 2,3 / 2)_{2}$ & - & 6p6f $(1 / 2,5 / 2)_{3}$ \\
\hline 2 & 2701.9136 & 36999.831 & -0.008 & 6p6d $(1 / 2,3 / 2)_{2}$ & - & $6 \mathrm{p} 6 \mathrm{f}(1 / 2,7 / 2)_{3}$ \\
\hline 7 & 2692.9308 & 37123.244 & 0.002 & 6p6d $(1 / 2,3 / 2)_{2}$ & - & 6p6f $(1 / 2,5 / 2)_{2}$ \\
\hline 5 & 2680.2723 & 37298.561 & -0.007 & $6 \mathrm{p} 7 \mathrm{~s}(3 / 2,1 / 2)_{1}$ & - & $6 \mathrm{p} 5 \mathrm{f}(3 / 2,7 / 2)_{2}$ \\
\hline 7 & 2630.9622 & 37997.579 & 0.000 & $6 \mathrm{p} 7 \mathrm{p}(1 / 2,1 / 2)_{1}$ & - & $6 \mathrm{p} 9 \mathrm{~d}(1 / 2,3 / 2)_{2}$ \\
\hline 11 & 2544.4103 & 39290.041 & -0.003 & $6 \mathrm{p} 7 \mathrm{~s}(1 / 2,1 / 2)_{1}$ & - & $6 \mathrm{p} 8 \mathrm{p}(1 / 2,3 / 2)_{2}$ \\
\hline 4 & 2530.4375 & 39506.982 & -0.004 & $6 \mathrm{p} 7 \mathrm{~s}(1 / 2,1 / 2)_{1}$ & - & $6 \mathrm{p} 8 \mathrm{p}(1 / 2,3 / 2)_{1}$ \\
\hline 2 & 2514.6745 & 39754.610 & & $6 \mathrm{p} 7 \mathrm{p}(1 / 2,3 / 2)_{2}$ & - & $6 \mathrm{p} 7 \mathrm{~d}(3 / 2,5 / 2)_{3}$ \\
\hline 5 & 2512.5739 & 39787.844 & 0.000 & $6 \mathrm{~s} \mathrm{p}^{3}{ }^{5} \mathrm{~S}_{2}$ & - & 6p6f $(1 / 2,5 / 2)_{3}$ \\
\hline 37 & 2502.8591 & 39942.269 & 0.001 & $6 \mathrm{~s} 6 \mathrm{p}^{3}{ }^{5} \mathrm{~S}_{2}$ & - & $6 \mathrm{p} 6 \mathrm{f}(1 / 2,7 / 2)_{3}$ \\
\hline 6 & 2501.0242 & 39971.571 & & $6 \mathrm{p} 7 \mathrm{~s}(1 / 2,1 / 2)_{0}$ & - & $6 \mathrm{p} 8 \mathrm{p}(1 / 2,3 / 2)_{1}$ \\
\hline 4 & 2495.1499 & 40065.670 & -0.001 & $6 \mathrm{~s} 6 \mathrm{p}^{3}{ }^{5} \mathrm{~S}_{2}$ & - & $6 \mathrm{p} 6 \mathrm{f}(1 / 2,5 / 2)_{2}$ \\
\hline 13 & 2480.1781 & 40307.512 & 0.000 & $6 \mathrm{p} 7 \mathrm{~s}(1 / 2,1 / 2)_{1}$ & - & $6 \mathrm{p} 7 \mathrm{p}(3 / 2,3 / 2)_{2}$ \\
\hline 6 & 2418.7166 & 41331.683 & & $6 \mathrm{p} 7 \mathrm{p}(1 / 2,1 / 2)_{1}$ & - & $6 \mathrm{p} 10 \mathrm{~d}(1 / 2,3 / 2)_{2}$ \\
\hline 54 & 2368.3838 & 42209.992 & -0.002 & $6 \mathrm{p}^{2}{ }^{1} \mathrm{D}_{2}$ & - & $6 \mathrm{~s} 6 \mathrm{p}^{3}{ }^{5} \mathrm{~S}_{2}$ \\
\hline 4 & 2325.2984 & 42992.034 & -0.014 & $6 \mathrm{p} 6 \mathrm{~d}(1 / 2,5 / 2)_{3}$ & - & $6 \mathrm{p} 8 \mathrm{f}(1 / 2,7 / 2)_{4}$ \\
\hline 3 & 2251.7291 & 44396.556 & -0.006 & $6 \mathrm{p} 6 \mathrm{~d}(1 / 2,5 / 2)_{3}$ & - & $6 \mathrm{p} 5 \mathrm{f}(3 / 2,5 / 2)_{4}$ \\
\hline 18 & 2214.0307 & 45152.425 & 0.002 & $6 \mathrm{p}^{2}{ }^{1} \mathrm{D}_{2}$ & - & $6 \mathrm{p} 6 \mathrm{~d}(1 / 2,3 / 2)_{2}$ \\
\hline 37 & 2186.9297 & 45711.907 & 0.000 & $6 \mathrm{p}^{2}{ }^{1} \mathrm{~S}_{0}$ & - & $6 \mathrm{p} 7 \mathrm{~s}(3 / 2,1 / 2)_{1}$ \\
\hline 5 & 2143.4827 & 46638.356 & 0.003 & $6 \mathrm{p}^{2}{ }^{1} \mathrm{D}_{2}$ & - & $6 \mathrm{p} 6 \mathrm{~d}(1 / 2,3 / 2)_{1}$ \\
\hline 200 & $1902.3422^{d}$ & 52566.777 & 0.000 & $6 \mathrm{p}^{2}{ }^{3} \mathrm{P}_{2}$ & - & $6 \mathrm{p} 7 \mathrm{~s}(1 / 2,1 / 2)_{1}$ \\
\hline 100 & $1791.842^{e}$ & 55808.49 & 0.000 & $6 \mathrm{p}^{2}{ }^{3} \mathrm{P}_{1}$ & - & $6 \mathrm{p} 7 \mathrm{~s}(1 / 2,1 / 2)_{0}$ \\
\hline 500 & $1436.8130^{e}$ & 69598.475 & 0.000 & $6 \mathrm{p}^{2}{ }^{3} \mathrm{P}_{0}$ & - & $6 \mathrm{p} 7 \mathrm{~s}(1 / 2,1 / 2)_{1}$ \\
\hline
\end{tabular}

${ }^{a}$ Signal-to-noise ratio of the strongest component. ${ }^{b}$ Centre of gravity air wavelength above $2000 \AA$ and vacuum wavelength below $2000 \AA .{ }^{c}$ Tentative identifiction, the energy levels in the transition can not be substantiated from other lines. ${ }^{d}$ Measured by Wahlgren et al. (1994). ${ }^{e}$ Measured by Wahlgren et al. (2001).

by comparing the hfs constants derived from all transitions involving a specific level. The criteria for deriving errors included considerations of line intensities, number of lines involving the levels, as well as line blending issues. A statistical approach was neglected since only one or a few lines were involved in the determination of the hfs constants of most levels, which could lead to erratic results in the uncertainty estimates. In cases where only one transition was observed, the uncertainty was estimated from the uncertainties of constants derived from lines with similar 
Table 2. Bi II even levels.

\begin{tabular}{|c|c|c|c|c|c|c|c|c|c|}
\hline \multirow[t]{2}{*}{ Designation } & \multirow[t]{2}{*}{$J$} & \multirow{2}{*}{$\begin{array}{l}\text { Energy } \\
\left(\mathrm{cm}^{-1}\right)\end{array}$} & \multirow{2}{*}{\multicolumn{2}{|c|}{$\begin{array}{c}\text { Eigenvector comp. }^{a} \\
(\%)\end{array}$}} & \multicolumn{2}{|c|}{ Hyperfine constants ${ }^{b}$} & \multicolumn{2}{|c|}{ Other exp. ${ }^{b}$} & \multirow[t]{2}{*}{ Ref. $^{c}$} \\
\hline & & & & & $A(\mathrm{mK})$ & & $A(\mathrm{mK})$ & $B(\mathrm{mK})$ & \\
\hline $6 \mathrm{p}^{2}{ }^{3} \mathrm{P}$ & 0 & $0.000^{d}$ & $83{ }^{3} \mathrm{P}$ & $17^{1} \mathrm{~S}$ & & & & & \\
\hline $6 \mathrm{p}^{2}{ }^{3} \mathrm{P}$ & 1 & $13325.401^{d}$ & $100{ }^{3} \mathrm{P}$ & & & & $-82.9(1)$ & $-16.5(10)$ & {$[1]$} \\
\hline & & & & & & & $-82.5(7)$ & $-15.5(35)$ & {$[2]$} \\
\hline & & & & & & & -82.85 & -15.7 & {$[3]$} \\
\hline $6 \mathrm{p}^{2}{ }^{3} \mathrm{P}$ & 2 & 17031.698 & $52{ }^{3} \mathrm{P}$ & $48^{1} \mathrm{D}$ & $112.6(3)$ & $-10(5)$ & $112.6(1)$ & $-8(1)$ & {$[1]$} \\
\hline & & & & & & & $115.1(28)$ & $-72(161)$ & {$[2]$} \\
\hline $6 \mathrm{p}^{2}{ }^{1} \mathrm{D}$ & 2 & 33938.718 & $52{ }^{1} \mathrm{D}$ & $48^{3} \mathrm{P}$ & $27.4(1)$ & $-24(2)$ & $27.4(2)$ & $-29(3)$ & {$[1]$} \\
\hline & & & & & & & $27.5(7)$ & $-12(16)$ & {$[2]$} \\
\hline & & & & & & & $27.18(11)$ & $-8.3(16)$ & [4] \\
\hline $6 \mathrm{p}^{2}{ }^{1} \mathrm{~S}$ & 0 & 44173.768 & $82{ }^{1} \mathrm{~S}$ & $17^{3} \mathrm{P}$ & & & & & \\
\hline $6 \mathrm{p} 7 \mathrm{p}(1 / 2,1 / 2)$ & 1 & 84280.446 & $100(1 / 2,1 / 2)$ & & $269.25(5)$ & $1(2)$ & $270.7(6)$ & $-0.6(18)$ & {$[5]$} \\
\hline $6 \mathrm{p} 7 \mathrm{p}(1 / 2,1 / 2)$ & 0 & 87078.777 & $98(1 / 2,1 / 2)$ & & & & & & \\
\hline $6 \mathrm{p} 7 \mathrm{p}(1 / 2,3 / 2)$ & 1 & 88566.685 & $100(1 / 2,3 / 2)$ & & $-102.10(5)$ & $-1(1)$ & $-102.5(3)$ & $-0.2(3)$ & {$[5]$} \\
\hline $6 p 7 p(1 / 2,3 / 2)$ & 2 & 88789.478 & $99(1 / 2,3 / 2)$ & & $123.35(10)$ & $-12(2)$ & 123.6 & $-6.2(21)$ & {$[5]$} \\
\hline $6 \mathrm{p} 7 \mathrm{p}(3 / 2,1 / 2)$ & 2 & 105085.532 & $50(3 / 2,1 / 2)$ & $475 \mathrm{f}(1 / 2,5 / 2)$ & $-13.6(3)$ & $-20(8)$ & & & \\
\hline $6 \mathrm{p} 7 \mathrm{p}(3 / 2,1 / 2)$ & 1 & 105270.947 & $85(3 / 2,1 / 2)$ & $13(3 / 2,3 / 2)$ & $13.3(8)$ & $-10(10)$ & $17.4(10)$ & $-2.1(21)$ & {$[5]$} \\
\hline $6 \mathrm{p} 5 \mathrm{f}(1 / 2,7 / 2)$ & 3 & 105289.732 & $82(1 / 2,7 / 2)$ & $137 \mathrm{p}(3 / 2,3 / 2)$ & $-28.8(2)$ & $-8(4)$ & & & \\
\hline $6 \mathrm{p} 5 \mathrm{f}(1 / 2,5 / 2)$ & 3 & 105451.045 & $95(1 / 2,5 / 2)$ & & $56.9(4)$ & $5(5)$ & & & \\
\hline $6 \mathrm{p} 5 \mathrm{f}(1 / 2,7 / 2)$ & 4 & 105728.060 & $100(1 / 2,7 / 2)$ & & $59.1(3)$ & $-6(5)$ & & & \\
\hline $6 \mathrm{p} 5 \mathrm{f}(1 / 2,5 / 2)$ & 2 & 106449.487 & $51(1 / 2,5 / 2)$ & $417 \mathrm{p}(3 / 2,1 / 2)$ & $-23.25(20)$ & $-13(5)$ & $-21.0(8)$ & $-12.9(83)$ & {$[5]$} \\
\hline $6 \mathrm{p} 7 \mathrm{p}(3 / 2,3 / 2)$ & 3 & 108280.653 & $86(3 / 2,3 / 2)$ & $135 \mathrm{f}(1 / 2,7 / 2)$ & $10.4(2)$ & $-40(10)$ & $11.3(4)$ & $-16.1(21)$ & {$[5]$} \\
\hline $6 \mathrm{p} 7 \mathrm{p}(3 / 2,3 / 2)$ & 1 & 108406.284 & $76(3 / 2,3 / 2)$ & $14(3 / 2,1 / 2)$ & $-13.7(4)$ & $8(5)$ & & & \\
\hline $6 \mathrm{p} 8 \mathrm{p}(1 / 2,3 / 2)$ & 2 & 108888.519 & $73(1 / 2,3 / 2)$ & $267 \mathrm{p}(3 / 2,3 / 2)$ & $101.4(4)$ & $6(5)$ & $101.8(12)$ & $1.1(35)$ & {$[5]$} \\
\hline $6 \mathrm{p} 8 \mathrm{p}(1 / 2,3 / 2)$ & 1 & 109105.461 & $92(1 / 2,3 / 2)$ & & $-69.8(3)$ & $-7(5)$ & & & \\
\hline $6 \mathrm{p} 7 \mathrm{p}(3 / 2,3 / 2)$ & 2 & 109905.987 & $69(3 / 2,3 / 2)$ & $228 \mathrm{p}(1 / 2,3 / 2)$ & $37.05(10)$ & $-8(3)$ & $37.2(6)$ & $-12.7(26)$ & {$[5]$} \\
\hline $6 \mathrm{p} 7 \mathrm{p}(3 / 2,3 / 2)$ & 0 & 112650.875 & $89(3 / 2,3 / 2)$ & $58 \mathrm{p}(1 / 2,1 / 2)$ & & & & & \\
\hline 6p6f $(1 / 2,5 / 2)$ & 3 & 115936.556 & $100(1 / 2,5 / 2)$ & & $78.95(20)$ & $-5(10)$ & & & \\
\hline 6p6f $(1 / 2,7 / 2)$ & 3 & 116090.980 & $99(1 / 2,7 / 2)$ & & $-57.75(10)$ & $25(3)$ & & & \\
\hline $6 \mathrm{p} 6 \mathrm{f}(1 / 2,7 / 2)$ & 4 & 116140.866 & $100(1 / 2,7 / 2)$ & & $59.5(5)$ & $40(20)$ & & & \\
\hline 6p6f $(1 / 2,5 / 2)$ & 2 & 116214.383 & $99(1 / 2,5 / 2)$ & & $-78.0(2)$ & $-2(8)$ & & & \\
\hline $6 \mathrm{p} 8 \mathrm{f}(1 / 2,5 / 2)$ & 3 & 125133.866 & $72(1 / 2,5 / 2)$ & $16(1 / 2,7 / 2)$ & $22.5(2)$ & $-9(5)$ & & & \\
\hline $6 \mathrm{p} 8 \mathrm{f}(1 / 2,7 / 2)$ & 4 & 125249.299 & $88(1 / 2,7 / 2)$ & $125 \mathrm{f}(3 / 2,7 / 2)$ & $51.6(8)$ & - & & & \\
\hline $6 \mathrm{p} 5 \mathrm{f}(3 / 2,5 / 2)$ & 3 & 125385.746 & $82(3 / 2,5 / 2)$ & $88 \mathrm{f}(1 / 2,5 / 2)$ & $19.2(3)$ & $7(6)$ & $15(6)$ & - & {$[6]$} \\
\hline $6 \mathrm{p} 5 \mathrm{f}(3 / 2,7 / 2)$ & 4 & 125536.550 & $72(3 / 2,7 / 2)$ & $18(3 / 2,5 / 2)$ & $11.2(3)$ & $0(10)$ & $5(10)$ & - & {$[6]$} \\
\hline $6 \mathrm{p} 5 \mathrm{f}(3 / 2,7 / 2)$ & 3 & 126107.642 & $84(3 / 2,7 / 2)$ & $9(3 / 2,5 / 2)$ & $-2.5(5)$ & $27(5)$ & $-11(3)$ & - & {$[6]$} \\
\hline $6 \mathrm{p} 5 \mathrm{f}(3 / 2,7 / 2)$ & 5 & $126266 .^{e}$ & $100(3 / 2,7 / 2)$ & & $-34(2)$ & $100(50)$ & & & \\
\hline $6 \mathrm{p} 5 \mathrm{f}(3 / 2,5 / 2)$ & 2 & 126299.205 & $91(3 / 2,5 / 2)$ & & $8.2(3)$ & $23(7)$ & 11 & - & {$[6]$} \\
\hline $6 \mathrm{p} 5 \mathrm{f}(3 / 2,5 / 2)$ & 4 & 126653.813 & $81(3 / 2,5 / 2)$ & $16(3 / 2,7 / 2)$ & $12.60(15)$ & $-36(5)$ & & & \\
\hline $6 \mathrm{p} 5 \mathrm{f}(3 / 2,5 / 2)$ & 1 & 127062.615 & $98(3 / 2,5 / 2)$ & & $-18.55(15)$ & $-4(2)$ & & & \\
\hline $6 \mathrm{p} 5 \mathrm{f}(3 / 2,7 / 2)$ & 2 & 127184.243 & $97(3 / 2,7 / 2)$ & & $-15.5(3)$ & $-18(5)$ & & & \\
\hline
\end{tabular}

${ }^{a}$ The two largest eigenvector components. The second component is shown only in cases where the largest components is smaller than $90 \%$. Configuration is shown for the second component when it is not the same as for the first component.

${ }^{b}$ Numbers in parenthesis represents an estimated fitting error for the last digit(s).

${ }^{c}$ References to previous $A$ and $B$ determinations: [1] Bouazza \& Bauche (1988); [2] Cole (1964); [3] George et al. (1985); [4] Arcimowicz \& Dembczynski (1979); [5] Grabowski et al. (1996); [6] Stachowska et al. (1987).

${ }^{d}$ The connection between the two lowest levels of the $6 \mathrm{p}^{2}$ ground configuration and the excited configurations is established by the lines at $1436 \AA$ and $1791 \AA$ reported by Wahlgren et al. (2001).

${ }^{e}$ Level value from the parametric fit. The hfs constants were derived from a line tentatively identified as the combination with $6 \mathrm{p} 6 \mathrm{~d}(3 / 2,5 / 2)_{4}$, but the latter level is not connected to the rest of the system. The uncertainty of the level is estimated to $\pm 400 \mathrm{~cm}^{-1}$, based on comparisons to Cowan calculations of other levels.

SNR and complexity. In the last columns of Tables 2 and 3 hfs constants from previous measurements are presented with their uncertainties and references.

All levels presented in AEL III (Moore 1958) have been confirmed by our data, except those at 107976 , 115990 and $117004 \mathrm{~cm}^{-1}$. These levels were observed by
Crawford \& McLay (1934), but transitions involving these levels can not be found in our spectra. The Cowan calculations in this paper predict energy levels with corresponding $J$ values near 107976 and $117004 \mathrm{~cm}^{-1}$, but fail to predict a level near $115990 \mathrm{~cm}^{-1}$. In the paper by Crawford \& McLay this level is listed as a tentative 
Table 3. Bi II odd levels.

\begin{tabular}{|c|c|c|c|c|c|c|c|c|c|}
\hline \multirow[t]{2}{*}{ Designation } & \multirow[t]{2}{*}{$J$} & \multirow{2}{*}{$\begin{array}{l}\text { Energy } \\
\left(\mathrm{cm}^{-1}\right)\end{array}$} & \multirow{2}{*}{\multicolumn{2}{|c|}{$\begin{array}{c}\text { Eigenvector comp. }^{a} \\
(\%)\end{array}$}} & \multicolumn{2}{|c|}{ Hyperfine constants ${ }^{b}$} & \multicolumn{2}{|c|}{ Other exp. ${ }^{b}$} & \multirow[t]{2}{*}{ Ref. $^{c}$} \\
\hline & & & & & $A(\mathrm{mK})$ & $B(\mathrm{mK})$ & $A(\mathrm{mK})$ & $B(\mathrm{mK})$ & \\
\hline $6 \mathrm{p} 7 \mathrm{~s}(1 / 2,1 / 2)$ & 0 & 69133.891 & $100(1 / 2,1 / 2)$ & & & & & & \\
\hline \multirow[t]{4}{*}{$6 \mathrm{p} 7 \mathrm{~s}(1 / 2,1 / 2)$} & 1 & 69598.475 & $98(1 / 2,1 / 2)$ & & $390.7(1)$ & $3(1)$ & $391.1(1)$ & $2.8(6)$ & {$[1]$} \\
\hline & & & & & & & $392.1(7)$ & $0.6(11)$ & {$[2]$} \\
\hline & & & & & & & 390.70 & 3.3 & {$[3]$} \\
\hline & & & & & & & $390.74(5)$ & $2.3(3)$ & {$[4]$} \\
\hline $6 \mathrm{~s} 6 \mathrm{p}^{3}{ }^{5} \mathrm{~S}$ & 2 & 76148.712 & $55{ }^{5} \mathrm{~S}$ & $18{ }^{3} \mathrm{P}$ & $401.30(5)$ & $-12(8)$ & $401.35(11)$ & $-9.4(16)$ & {$[5]$} \\
\hline $6 \mathrm{p} 6 \mathrm{~d}(1 / 2,3 / 2)$ & 2 & 79091.141 & $97(1 / 2,3 / 2)$ & & $119.5(5)$ & $0(5)$ & & & \\
\hline $6 \mathrm{p} 6 \mathrm{~d}(1 / 2,3 / 2)$ & 1 & 80577.071 & $73(1 / 2,3 / 2)$ & $13 \mathrm{p}^{33} \mathrm{D}$ & $-174.1(2)$ & $-5(2)$ & & & \\
\hline $6 \mathrm{p} 6 \mathrm{~d}(1 / 2,5 / 2)$ & 2 & 82049.632 & $71(1 / 2,5 / 2)$ & $26 \mathrm{p}^{35} \mathrm{~S}$ & $90.75(20)$ & $10(2)$ & & & \\
\hline $6 \mathrm{p} 6 \mathrm{~d}(1 / 2,5 / 2)$ & 3 & 82257.251 & $94(1 / 2,5 / 2)$ & & $84.0(2)$ & $0(4)$ & & & \\
\hline \multirow[t]{2}{*}{$6 \mathrm{p} 7 \mathrm{~s}(3 / 2,1 / 2)$} & 2 & 88771.443 & $99(3 / 2,1 / 2)$ & & $106.0(2)$ & $-38(3)$ & $106.0(1)$ & $-38(1)$ & {$[1]$} \\
\hline & & & & & & & $107.8(3)$ & $-34.5(29)$ & {$[2]$} \\
\hline \multirow[t]{3}{*}{$6 \mathrm{p} 7 \mathrm{~s}(3 / 2,1 / 2)$} & 1 & 89885.675 & $84(3 / 2,1 / 2)$ & $66 \mathrm{~d}(3 / 2,5 / 2)$ & $-60.6(1)$ & $-22(2)$ & $-60.6(1)$ & $-21(1)$ & [1] \\
\hline & & & & & & & $-61.4(8)$ & $-25.9(30)$ & {$[2]$} \\
\hline & & & & & & & $-60.71(50)$ & $-21.5(5)$ & {$[4]$} \\
\hline $6 s 6 p^{3}{ }^{3} \mathrm{D}$ & 1 & 94442.227 & $44^{3} \mathrm{D}$ & $226 \mathrm{~d}(1 / 2,3 / 2)$ & $-307.9(5)$ & $-23(10)$ & $-305(1)$ & $-50(4)$ & {$[6]$} \\
\hline $6 s 6 p^{3}{ }^{3} \mathrm{D}$ & 2 & 94930.640 & $48^{3} \mathrm{D}$ & $316 \mathrm{~d}(3 / 2,3 / 2)$ & $76.5(5)$ & $-15(10)$ & $70(10)$ & $-36(1)$ & [6] \\
\hline $6 \mathrm{~s} 6 \mathrm{p}^{3}{ }^{3} \mathrm{D}$ & 3 & 96062.356 & $50{ }^{3} \mathrm{D}$ & $316 \mathrm{~d}(3 / 2,5 / 2)$ & $201.3(2)$ & $-9(5)$ & $196(8)$ & & {$[6]$} \\
\hline $6 \mathrm{p} 6 \mathrm{~d}(3 / 2,5 / 2)$ & 2 & 99011.328 & $55(3 / 2,5 / 2)$ & $19(3 / 2,3 / 2)$ & $108.9(4)$ & $-12(6)$ & & & \\
\hline $6 \mathrm{p} 6 \mathrm{~d}(3 / 2,5 / 2)$ & 4 & $99405^{d}$ & $100(3 / 2,5 / 2)$ & & $9(2)$ & $11(20)$ & & & \\
\hline $6 \mathrm{p} 6 \mathrm{~d}(3 / 2,3 / 2)$ & 0 & 100182.446 & $72(3 / 2,3 / 2)$ & $18 \mathrm{p}^{3}{ }^{3} \mathrm{P}$ & & & & & \\
\hline $6 \mathrm{p} 6 \mathrm{~d}(3 / 2,3 / 2)$ & 1 & 100494.931 & $64(3 / 2,3 / 2)$ & $126 \mathrm{~d}(3 / 2,5 / 2)$ & $108.4(3)$ & $11(4)$ & & & \\
\hline $6 \mathrm{p} 8 \mathrm{~s}(1 / 2,1 / 2)$ & 1 & 101340.430 & $86(1 / 2,1 / 2)$ & $116 \mathrm{~d}(3 / 2,3 / 2)$ & $279.8(8)$ & $6(10)$ & & & \\
\hline $6 \mathrm{p} 8 \mathrm{~s}(1 / 2,1 / 2)$ & 0 & 101463.435 & $90(1 / 2,1 / 2)$ & $106 \mathrm{~d}(3 / 2,3 / 2)$ & & & & & \\
\hline $6 \mathrm{p} 6 \mathrm{~d}(3 / 2,3 / 2)$ & 2 & 102331.668 & $38(3 / 2,5 / 2)$ & $34(3 / 2,3 / 2)$ & $100.3(5)$ & $8(5)$ & & & \\
\hline $6 \mathrm{p} 6 \mathrm{~d}(3 / 2,3 / 2)$ & 3 & 103003.505 & $59(3 / 2,3 / 2)$ & $287 \mathrm{~d}(1 / 2,5 / 2)$ & $79.1(3)$ & $-30(5)$ & & & \\
\hline $6 \mathrm{p} 7 \mathrm{~d}(1 / 2,3 / 2)$ & 2 & 105527.197 & $95(1 / 2,3 / 2)$ & & $116.2(2)$ & $5(3)$ & & & \\
\hline $6 \mathrm{p} 6 \mathrm{~d}(3 / 2,5 / 2)$ & 1 & $106611.224^{e}$ & $64(3 / 2,5 / 2)$ & $137 \mathrm{~d}(1 / 2,3 / 2)$ & & & & & \\
\hline $6 \mathrm{p} 6 \mathrm{~d}(3 / 2,5 / 2)$ & 3 & 108009.057 & $41(3 / 2,5 / 2)$ & $367 \mathrm{~d}(1 / 2,5 / 2)$ & $56.3(4)$ & $-25(10)$ & & & \\
\hline $6 \mathrm{p} 7 \mathrm{~d}(1 / 2,3 / 2)$ & 1 & 108126.733 & $75(1 / 2,3 / 2)$ & $86 \mathrm{~d}(3 / 2,5 / 2)$ & $-20.9(7)$ & $0(10)$ & & & \\
\hline $6 \mathrm{p} 7 \mathrm{~d}(1 / 2,5 / 2)$ & 2 & $109158.128^{e}$ & $34(1 / 2,5 / 2)$ & $31 \mathrm{p}^{33} \mathrm{D}$ & & & $170(6)$ & $10(10)$ & {$[6]$} \\
\hline $6 \mathrm{p} 9 \mathrm{~s}(1 / 2,1 / 2)$ & 0 & 114579.101 & $87(1 / 2,1 / 2)$ & $11 \mathrm{p}^{3}{ }^{3} \mathrm{P}$ & & & & & \\
\hline $6 p 9 s(1 / 2,1 / 2)$ & 1 & 114613.372 & $76(1 / 2,1 / 2)$ & $9 \mathrm{p}^{3}{ }^{3} \mathrm{P}$ & $218.15(10)$ & $-18(1)$ & & & \\
\hline $6 \mathrm{p} 8 \mathrm{~d}(1 / 2,5 / 2)$ & 2 & 116942.813 & $49(1 / 2,5 / 2)$ & $40(1 / 2,3 / 2)$ & $35.65(20)$ & $5(3)$ & & & \\
\hline $6 \mathrm{p} 8 \mathrm{~d}(1 / 2,5 / 2)$ & 3 & 117224.556 & $96(1 / 2,5 / 2)$ & & $71.3(3)$ & $5(10)$ & & & \\
\hline $6 \mathrm{p} 9 \mathrm{~d}(1 / 2,3 / 2)$ & 2 & 122278.025 & $89(1 / 2,3 / 2)$ & & $117.3(5)$ & $-30(10)$ & & & \\
\hline $6 p 10 d(1 / 2,3 / 2)$ & 2 & 125612.129 & $90(1 / 2,3 / 2)$ & & $100.0(5)$ & $-10(5)$ & & & \\
\hline $6 \mathrm{p} 7 \mathrm{~d}(3 / 2,3 / 2)$ & 3 & 126949.444 & $72(3 / 2,3 / 2)$ & $26(3 / 2,5 / 2)$ & $23.6(3)$ & $-21(6)$ & & & \\
\hline $6 \mathrm{p} 7 \mathrm{~d}(3 / 2,5 / 2)$ & 3 & 128544.088 & $71(3 / 2,5 / 2)$ & $26(3 / 2,3 / 2)$ & $-31(1)$ & & & & \\
\hline
\end{tabular}

${ }^{a}$ The two largest eigenvector components. The second component is shown only in cases where the largest components is smaller than $90 \%$. Configuration is shown for the second component when it is not the same as for the first component.

${ }^{b}$ Numbers in parenthesis represents an estimated fitting error for the last digit(s).

${ }^{c}$ References to previous $A$ and $B$ determinations: [1] Bouazza \& Bauche (1988); [2] Grabowski et al. (1996); [3] George et al. (1985); [4] Eisele et al. (1968); [5] Arcimowicz \& Dembczynski (1979); [6] Stachowska et al. (1987).

${ }^{d}$ Level value from the parametric fit. The hfs constants were derived from a line tentatively identified as the combination with $6 \mathrm{p} 5 \mathrm{f}(3 / 2,7 / 2)_{5}$, but the latter level is not connected to the rest of the system. The uncertainty of the level is estimated to $\pm 400 \mathrm{~cm}^{-1}$, based on comparisons to Cowan calculations of other levels.

${ }^{e}$ Level value derived from VUV lines reported in Wahlgren et al. (2001). No combinations observed in the present work.

identification from one weak spectral feature. Two other AEL levels, the odd levels at 106611 and $109158 \mathrm{~cm}^{-1}$, have not been confirmed in the present work, but as they are confirmed by combinations reported by Wahlgren et al. (2001), they are included in Table 3 . The $J$ values of two levels $(105270,106449)$ have been changed. Five levels established by Stachowska et al. (1987) have also been confirmed, but the $J$ of the level at $125536 \mathrm{~cm}^{-1}$ has been changed from 2 to 4 . The changes of $J$ values are based on the hfs analysis and the theoretical interpretation of the level structure. 


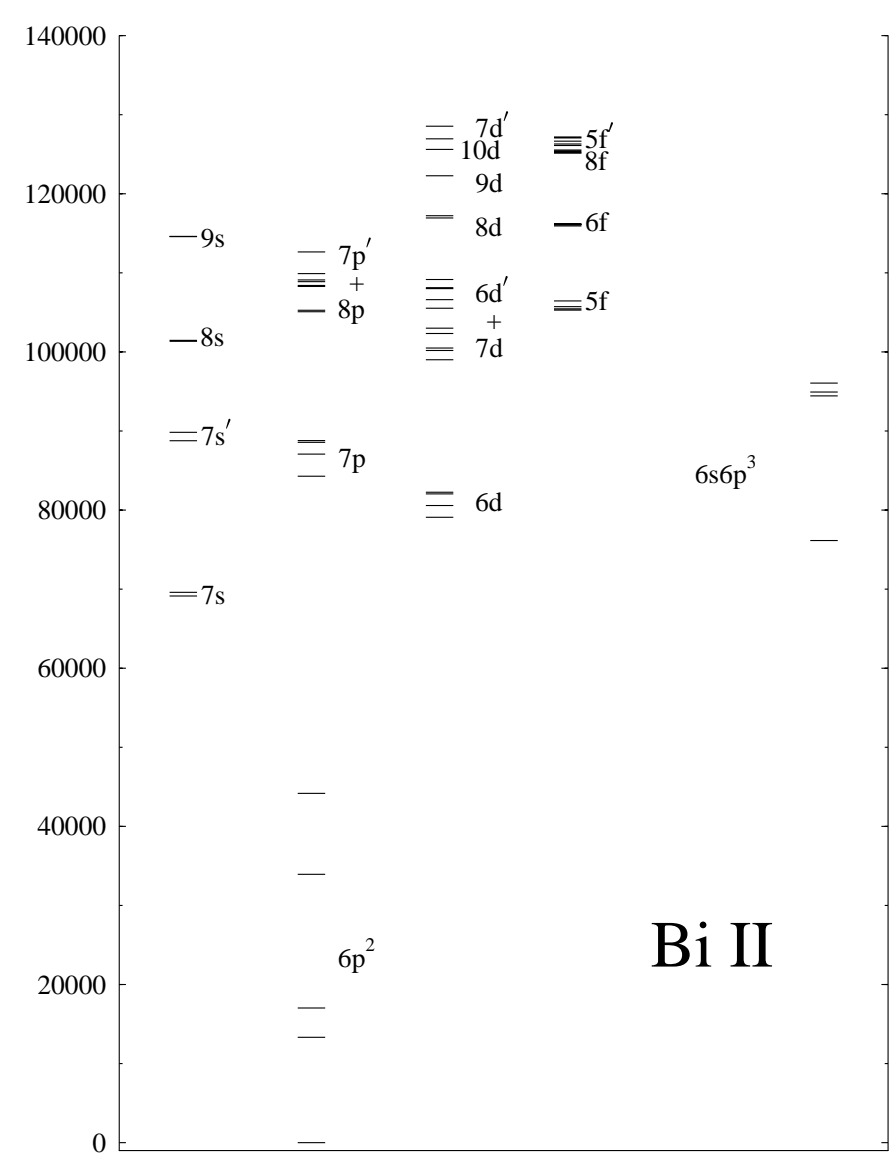

Fig. 2. An overview of the energy level system of Bi II.

Identification of a number of previously unidentified lines have enabled the establishment of 17 new levels. Some of the high levels are derived from only one line, but in these cases the identity of the lower level can be unambiguously established by means of the hyperfine constants derived from the analysis of the hfs pattern.

Two new levels, the $J=4$ level of $6 \mathrm{p} 6 \mathrm{~d}$ and the $J=5$ level of $6 \mathrm{p} 5 \mathrm{f}$, have not been connected to the rest of the system by any observed line. A tentative identification of a line representing the transition between the two levels is given in Table 1. This identification is based on the position of the line predicted by the theoretical calculations described below, and on the observed hfs pattern, consistent with the pattern expected for a $J=4 \Leftrightarrow 5$ transition. In fact, it is the only line with hyperfine structure observed in our work that can not be identified in any other way. The connection of the two levels to the rest of the system is difficult, as they are the highest $J$ levels of each configuration, and the lower level, $J=4$ of $6 \mathrm{p} 6 \mathrm{~d}$, is metastable.

\section{A theoretical study of the Bill energy level structure}

The ground configuration of $\mathrm{Bi} I \mathrm{II}$ is $6 \mathrm{p}^{2}$ and the excited configurations belong to the $6 \mathrm{p} n l$ system (see Fig. 2). Also, the inner-shell excited configuration $6 \mathrm{~s} 6 \mathrm{p}^{3}$ is situated below the first ionization limit. As expected for the heaviest elements in the periodic table, the level structure of Bi II can not be well described by the $L S$ coupling approximation. The magnetic spin-orbit interaction is large, giving a fine structure splitting of the $6 \mathrm{p}^{2} \mathrm{P}$ parent term of $20788 \mathrm{~cm}^{-1}$. This could be compared to the corresponding parent splitting of the homologous N II system, amounting to $174 \mathrm{~cm}^{-1}$. This means that the structure can be represented by the $j j$ coupling approximation, and the level designations used in this work are based on this coupling. The designations have the form $6 \mathrm{p} n l\left(j_{6 \mathrm{p}}, j_{n l}\right)_{J}$.

The large parent splitting gives rise to a particular complication in the interpretation of the structure, as each $6 \mathrm{p} n l$ configuration is split in two groups, separated by a distance equal to the parent splitting. As this splitting is of the same magnitude or larger than the distance between the average energies of adjacent configurations, the configurations will overlap, and will in certain regions be strongly mixed. This can be seen in Fig. 2, where the lower and the upper group of levels in each configuration are given the labels $n l$ and $n l^{\prime}$. This corresponds to $j_{6 \mathrm{p}}=1 / 2$ and $j_{6 \mathrm{p}}=3 / 2$ in the $j j$ notation.

In the present work the observed structure has been interpreted by the set of computer programs known as the Cowan code (Cowan 1981). An ab inito calculation with Hartree-Fock wave functions was followed by a parametric study, where significant energy parameters were fitted to the observed levels. All electrostatic energy integrals were scaled to $80 \%$ of the Hartree-Fock values in the ab inito calculation, as this is known to improve the agreement with the observed structure (Cowan 1981). This scaling was maintained for the parameters that were fixed during the parametric fit. The major eigenvector components of the observed levels, derived from the parametric calculations, are shown in Tables 2 and 3.

\subsection{The even configurations}

The calculations comprised the configurations $6 \mathrm{p}^{2}, 6 \mathrm{p} 7 \mathrm{p}$, $6 \mathrm{p} 8 \mathrm{p}$, and $6 \mathrm{p} 5 \mathrm{f}-6 \mathrm{p} 8 \mathrm{f}$. As the purity of the levels of the $6 \mathrm{p}^{2}$ ground configuration is the same in $L S$ and $j j$ coupling, we have chosen to use the more familiar $L S$ designation for these levels. The strongest configuration mixings, caused by complete overlap, appear between $6 \mathrm{p} 7 \mathrm{p}$ and $6 \mathrm{p} 8 \mathrm{p}$ and between $6 \mathrm{p} 7 \mathrm{p}$ and $6 \mathrm{p} 5 \mathrm{f}$. The Hartree-Fock and the fitted parameter values are shown in Table 4 . All parameters of $6 \mathrm{p} 7 \mathrm{f}$ were fixed at $100 \%$ or $80 \%$ of the Hartree-Fock values, as no levels of this configuration were observed. This is probably due to the fact that these levels are predicted to appear in a region where no enhancement due to charge and energy exchange in the light source is expected.

All configuration interaction parameters representing interactions other than these mentioned above are not shown in the table, since they are considered to be of peripheral interest. They were fixed at $80 \%$ of the HartreeFock values. 
Table 4. Fitted and Hartree-Fock energy parameters for the even Bi II configurations $\left(\mathrm{cm}^{-1}\right)$. Standard deviation of fit to observed levels $78 \mathrm{~cm}^{-1}$.

\begin{tabular}{|c|c|c|c|c|}
\hline Parameter & \multicolumn{2}{|c|}{ Fitted } & $\mathrm{HF}$ & Fitted/HF \\
\hline \multicolumn{5}{|l|}{$6 \mathrm{p}^{2}$} \\
\hline$E_{\mathrm{av}}$ & 22726 & \pm 41 & & \\
\hline$F^{2}(\mathrm{pp})$ & 29254 & \pm 376 & 38298 & 0.76 \\
\hline$\alpha$ & -63 & \pm 38 & & \\
\hline $\begin{array}{l}\zeta_{6 \mathrm{p}} \\
6 \mathrm{p} 7 \mathrm{p}\end{array}$ & 11808 & \pm 49 & 11682 & 1.01 \\
\hline$E_{\mathrm{av}}$ & 101067 & \pm 32 & & \\
\hline$\zeta_{6 \mathrm{p}}$ & 13475 & \pm 40 & 13504 & 1.00 \\
\hline$\zeta_{7 \mathrm{p}}$ & 2074 & \pm 54 & 1853 & 1.12 \\
\hline$F^{2}\left(\mathrm{pp}^{\prime}\right)$ & 7406 & \pm 338 & 9004 & 0.82 \\
\hline$G^{0}\left(\mathrm{pp}^{\prime}\right)$ & 1442 & \pm 41 & 2350 & 0.61 \\
\hline$G^{2}\left(\mathrm{pp}^{\prime}\right)$ & 1643 & \pm 288 & 2665 & 0.62 \\
\hline \multicolumn{5}{|l|}{$6 \mathrm{p} 8 \mathrm{p}$} \\
\hline$E_{\mathrm{av}}$ & 122017 & \pm 58 & & \\
\hline$\zeta_{6 \mathrm{p}}$ & 13443 & \pm 70 & 13575 & 0.99 \\
\hline$\zeta_{8 \mathrm{p}}$ & 755 & & 755 & $1.00^{a}$ \\
\hline$F^{2}\left(\mathrm{pp}^{\prime}\right)$ & 2676 & & 3345 & $0.80^{b}$ \\
\hline$G^{0}\left(\mathrm{pp}^{\prime}\right)$ & 623 & \pm 41 & 779 & $0.80^{b}$ \\
\hline$G^{2}\left(\mathrm{pp}^{\prime}\right)$ & 765 & & 956 & $0.80^{b}$ \\
\hline \multicolumn{5}{|l|}{$6 \mathrm{p} 5 \mathrm{f}$} \\
\hline$E_{\mathrm{av}}$ & 119341 & \pm 28 & & \\
\hline$\zeta_{6 \mathrm{p}}$ & 13633 & \pm 42 & 13534 & 1.01 \\
\hline$\zeta_{5 f}$ & 1.7 & & 2 & $1.00^{a}$ \\
\hline$F^{2}(\mathrm{pf})$ & 5850 & \pm 297 & 6289 & 0.93 \\
\hline$G^{2}(\mathrm{pf})$ & 1469 & \pm 416 & 2418 & 0.61 \\
\hline$G^{4}(\mathrm{pf})$ & 1266 & & 9961 & $0.80^{b}$ \\
\hline \multicolumn{5}{|l|}{$6 \mathrm{p} 6 \mathrm{f}$} \\
\hline$E_{\mathrm{av}}$ & 129676 & \pm 39 & & \\
\hline$\zeta_{6 \mathrm{p}}$ & 13566 & & 13566 & $1.00^{a}$ \\
\hline$\zeta_{6 \mathrm{f}}$ & 1.4 & & 1 & $1.00^{a}$ \\
\hline$F^{2}(\mathrm{pf})$ & 2536 & & 3170 & $0.80^{b}$ \\
\hline$G^{2}(\mathrm{pf})$ & 1305 & & 1631 & $0.80^{b}$ \\
\hline$G^{4}(\mathrm{pf})$ & 862 & & 1076 & $0.80^{a}$ \\
\hline \multicolumn{5}{|l|}{$6 \mathrm{p} 7 \mathrm{f}$} \\
\hline$E_{\mathrm{av}}$ & $135408^{a}$ & & & \\
\hline$\zeta_{6 \mathrm{p}}$ & 13590 & & 13590 & $1.00^{a}$ \\
\hline$\zeta_{7 \mathrm{f}}$ & 0.9 & & 0.9 & $1.00^{a}$ \\
\hline$F^{2}(\mathrm{pf})$ & 1454 & & 1818 & $0.80^{b}$ \\
\hline$G^{2}(\mathrm{pf})$ & 835 & & 1044 & $0.80^{b}$ \\
\hline$G^{4}(\mathrm{pf})$ & 554 & & 693 & $0.80^{a}$ \\
\hline \multicolumn{5}{|l|}{$6 \mathrm{p} 8 \mathrm{f}$} \\
\hline$E_{\mathrm{av}}$ & 138866 & \pm 10 & & \\
\hline$\zeta_{6 \mathrm{p}}$ & 13605 & & 13605 & $1.00^{a}$ \\
\hline$\zeta_{8 \mathrm{f}}$ & 0.6 & & 0.6 & $1.00^{a}$ \\
\hline$F^{2}(\mathrm{pf})$ & 908 & & 1135 & $0.80^{b}$ \\
\hline$G^{2}(\mathrm{pf})$ & 551 & & 689 & $0.80^{b}$ \\
\hline$G^{4}(\mathrm{pf})$ & 367 & & 459 & $0.80^{a}$ \\
\hline \multicolumn{5}{|l|}{ 6p7p-6p8p } \\
\hline$R^{2}\left(\mathrm{pp}^{\prime}, \mathrm{pp}^{\prime \prime}\right)$ & 2611 & \pm 497 & 4070 & $0.64^{c}$ \\
\hline$R^{0}\left(\mathrm{pp}^{\prime}, \mathrm{p}^{\prime \prime} \mathrm{p}\right)$ & 862 & \pm 164 & 1345 & $0.64^{c}$ \\
\hline$R^{2}\left(\mathrm{pp}^{\prime}, \mathrm{p}^{\prime \prime} \mathrm{p}\right)$ & 1013 & \pm 193 & 1580 & $0.64^{c}$ \\
\hline \multicolumn{5}{|l|}{$6 \mathrm{p} 7 \mathrm{p}-6 \mathrm{p} 5 \mathrm{f}$} \\
\hline$R^{2}\left(\mathrm{pp}^{\prime}, \mathrm{pf}\right)$ & 4142 & \pm 265 & 4118 & $1.01^{c}$ \\
\hline$R^{2}\left(\mathrm{pp}^{\prime}, \mathrm{fp}\right)$ & -712 & \pm 164 & -708 & $1.01^{c}$ \\
\hline
\end{tabular}

${ }^{a}$ Parameter fixed at Hartree-Fock value. ${ }^{b}$ Parameter fixed at $80 \%$ of Hartree-Fock value. ${ }^{c}$ Ratio of CI parameters involving the same pair of configurations fixed to their HF ratio.

\subsection{The odd configurations}

The configurations 6p7s, 6p8s, 6p9s, 6p6d, 6p7d, 6p8d and $6 \mathrm{~s} 6 \mathrm{p}^{3}$ were included in the calculation. For the same reason as mentioned for $6 \mathrm{p}^{2}, L S$ designations were used for $6 \mathrm{~s} 6 \mathrm{p}^{3}$. The configuration mixing is still more important for the odd configurations than for the even, as seen from the large values of the interaction parameters shown at the end of Table 5. 6p6d is strongly mixed with $6 \mathrm{p} 7 \mathrm{~s}$, $6 \mathrm{p} 7 \mathrm{~d}$ and $6 \mathrm{~s} 6 \mathrm{p}^{3}$, and there is also a considerable mixing between $6 \mathrm{p} 7 \mathrm{~d}$ and $6 \mathrm{~s}^{2} \mathrm{p}^{3}$. The configuration interaction parameters not shown in the table were fixed at $80 \%$ of the Hartree-Fock values.

In a separate ab initio calculation $6 \mathrm{p} 9 \mathrm{~d}$ and $6 \mathrm{p} 10 \mathrm{~d}$ were added to the set of configurations. As a result of this calculation the levels established at $122278 \mathrm{~cm}^{-1}$ and $125612 \mathrm{~cm}^{-1}$ were tentatively identified as $6 \mathrm{p} 9 \mathrm{~d}(1 / 2,3 / 2)_{2}$ and $6 \mathrm{p} 10 \mathrm{~d}(1 / 2,3 / 2)_{2}$.

\section{6. $\mathrm{Bi}$ II in HR 7775}

HR 7775 (= HD $193452=\beta^{2}$ Cap, $m_{v}=6.10$, B9.5 III-IVp $\mathrm{Hg})$ is a slowly rotating $\left(v \sin i=2 \mathrm{kms}^{-1}\right)$ HgMn star. HR 7775 has previously been analysed by Adelman (1994) and Wahlgren et al. (2000) in the optical region, and by Smith \& Dworetsky (1993) in the ultraviolet. The stellar parameters adopted in this work were $T_{\text {eff }}=10750 \mathrm{~K}, \log g=4.0, v \sin i=2 \mathrm{~km} \mathrm{~s}^{-1}$, and turbulent velocity $=0 \mathrm{kms}^{-1}$, as used by Wahlgren et al. (2000). The spectra of HR 7775 were obtained between July 2-13 1998 with the 2.56 m Nordic Optical Telescope (NOT) utilizing the SOviet-FINnish (SOFIN) echelle spectrograph (Tuominen et al. 1998; Ilyin 2000). The SOFIN spectrograph was mounted with a camera of focal length $f=1000 \mathrm{~mm}$ (camera 2), providing a resulting resolving power of approximately $R=80000$. In a recent investigation of HR 7775 (Wahlgren et al. 2000), it was noted that a large number of unidentified features could be found in the optical spectral region. Several of these features coincided in wavelength with $\mathrm{Bi}$ II lines observed in the laboratory analysis by Crawford \& McLay (1934). The identification of these features as Bi II lines seemed likely since the abundance of bismuth in HR 7775, as determined from ultraviolet Bi II lines, is greatly enhanced (Jacobs \& Dworetsky 1982; Wahlgren et al. 2001).

In the present investigation $14 \mathrm{Bi}$ II transitions have been detected in the optical region of HR 7775. The wavelength and identification of the observed features are presented in Table 6 along with the observed intensity and a theoretical or astrophysical log $g f$ value. Several of the Bi II features in HR 7775 are observed as either substantially broadened or as multiple spectral features, indicating the presence of noticeable hfs.

The bismuth abundance in HR 7775 and the astrophysical $g f$ values were determined by comparing the observed spectrum to a synthetic spectrum generated with the SYNTHE (Kurucz \& Avrett 1981; Kurucz 1993) program. Atomic line data were taken from the lists 
Table 5. Fitted and Hartree-Fock energy parameters for the odd Bi II configurations $\left(\mathrm{cm}^{-1}\right)$. Standard deviation of fit to observed levels $137 \mathrm{~cm}^{-1}$.

\begin{tabular}{|c|c|c|c|c|}
\hline Parameter & \multicolumn{2}{|c|}{ Fitted } & $\mathrm{HF}$ & Fitted/HF \\
\hline \multicolumn{5}{|l|}{$6 \mathrm{p} 7 \mathrm{~s}$} \\
\hline$E_{\mathrm{av}}$ & 82931 & \pm 96 & & \\
\hline$\zeta_{6 \mathrm{p}}$ & 13042 & \pm 97 & 13091 & 1.00 \\
\hline$G^{1}(\mathrm{ps})$ & 3855 & & 4819 & $0.80^{a}$ \\
\hline \multicolumn{5}{|l|}{$6 \mathrm{p} 8 \mathrm{~s}$} \\
\hline$E_{\mathrm{av}}$ & 115033 & \pm 115 & & \\
\hline$\zeta_{6 \mathrm{p}}$ & 13481 & & 13481 & $1.00^{b}$ \\
\hline$G^{1}(\mathrm{ps})$ & 973 & & 1216 & $0.80^{a}$ \\
\hline \multicolumn{5}{|l|}{$6 \mathrm{p} 9 \mathrm{~s}$} \\
\hline$E_{\mathrm{av}}$ & 127935 & \pm 248 & & \\
\hline$\zeta_{6 \mathrm{p}}$ & 13566 & & 13566 & $1.00^{b}$ \\
\hline$G^{1}(\mathrm{ps})$ & 421 & & 526 & $0.80^{a}$ \\
\hline \multicolumn{5}{|l|}{ 6p6d } \\
\hline$E_{\mathrm{av}}$ & 95687 & \pm 123 & & \\
\hline$\zeta_{6 \mathrm{p}}$ & 13143 & \pm 119 & 13015 & 1.00 \\
\hline$\zeta_{6 \mathrm{~d}}$ & 250 & & 250 & $1.00^{b}$ \\
\hline$F^{2}(\mathrm{pd})$ & 10406 & \pm 1323 & 18156 & 0.57 \\
\hline$G^{1}(\mathrm{pd})$ & 11364 & \pm 380 & 15918 & 0.71 \\
\hline$G^{3}(\mathrm{pd})$ & 6456 & \pm 1542 & 9961 & 0.65 \\
\hline \multicolumn{5}{|l|}{$6 \mathrm{p} 7 \mathrm{~d}$} \\
\hline$E_{\mathrm{av}}$ & 120129 & \pm 87 & & \\
\hline$\zeta_{6 \mathrm{p}}$ & 13521 & \pm 107 & 13463 & 1.00 \\
\hline$\zeta_{6 \mathrm{~d}}$ & 97 & & 97 & $1.00^{b}$ \\
\hline$F^{2}(\mathrm{pd})$ & 4369 & & 5461 & $0.80^{a}$ \\
\hline$G^{1}(\mathrm{pd})$ & 3421 & & 4276 & $0.80^{a}$ \\
\hline$G^{3}(\mathrm{pd})$ & 2246 & & 2808 & $0.80^{a}$ \\
\hline \multicolumn{5}{|l|}{$6 \mathrm{p} 8 \mathrm{~d}$} \\
\hline$E_{\mathrm{av}}$ & 130338 & \pm 111 & & \\
\hline$\zeta_{6 \mathrm{p}}$ & 13558 & & 13558 & $1.00^{b}$ \\
\hline$\zeta_{6 \mathrm{~d}}$ & 49 & & 49 & $1.00^{b}$ \\
\hline$F^{2}(\mathrm{pd})$ & 1984 & & 2480 & $0.80^{a}$ \\
\hline$G^{1}(\mathrm{pd})$ & 1524 & & 1905 & $0.80^{a}$ \\
\hline$G^{3}(\mathrm{pd})$ & 1018 & & 1273 & $0.80^{a}$ \\
\hline \multicolumn{5}{|l|}{$6 \mathrm{~s} 6 \mathrm{p}^{3}$} \\
\hline$E_{\mathrm{av}}$ & 108290 & \pm 902 & & \\
\hline$F^{2}(\mathrm{pp})$ & 31353 & \pm 3783 & 38594 & 0.81 \\
\hline$\alpha$ & -460 & \pm 272 & & \\
\hline$\zeta_{6 \mathrm{p}}$ & 12687 & \pm 675 & 11732 & 1.08 \\
\hline$G^{1}(\mathrm{sp})$ & 30585 & \pm 2046 & 48748 & 0.63 \\
\hline \multicolumn{5}{|l|}{$6 \mathrm{p} 7 \mathrm{~s}-6 \mathrm{p} 6 \mathrm{~d}$} \\
\hline$R^{2}(\mathrm{ps}, \mathrm{pd})$ & -11371 & \pm 1248 & -12603 & $0.90^{c}$ \\
\hline$R^{1}(\mathrm{ps}, \mathrm{dp})$ & -5391 & \pm 592 & -5975 & $0.90^{c}$ \\
\hline \multicolumn{5}{|l|}{$6 \mathrm{p} 6 \mathrm{~d}-6 \mathrm{p} 7 \mathrm{~d}$} \\
\hline$R^{2}(\mathrm{pd}, \mathrm{pd})$ & 4786 & \pm 365 & 7210 & $0.66^{c}$ \\
\hline$R^{1}(\mathrm{pd}, \mathrm{dp})$ & 5302 & \pm 408 & 8051 & $0.66^{c}$ \\
\hline$R^{3}(\mathrm{pd}, \mathrm{dp})$ & 3399 & \pm 261 & 5160 & $0.66^{c}$ \\
\hline \multicolumn{5}{|l|}{$6 \mathrm{p} 6 \mathrm{~d}-6 \mathrm{~s} 6 \mathrm{p}^{3}$} \\
\hline$R^{1}(\mathrm{sd}, \mathrm{pp})$ & 15069 & \pm 299 & 24725 & 0.61 \\
\hline \multicolumn{5}{|l|}{$6 \mathrm{p} 7 \mathrm{~d}-6 \mathrm{~s} 6 \mathrm{p}^{3}$} \\
\hline$R^{1}(\mathrm{sd}, \mathrm{pp})$ & 9985 & \pm 676 & 13240 & 0.75 \\
\hline
\end{tabular}

${ }^{a}$ Parameter fixed at $80 \%$ of Hartree-Fock value. ${ }^{b}$ Parameter fixed at Hartree-Fock value. ${ }^{c}$ Ratio of CI parameters involving the same pair of configurations fixed to their HF ratio.
Table 6. Observed Bi II lines in HR 7775.

\begin{tabular}{|c|c|c|c|c|c|}
\hline \multirow[t]{2}{*}{$\lambda(\AA)$} & \multirow[t]{2}{*}{$\operatorname{Int}^{a}$} & \multicolumn{2}{|c|}{ Energy levels } & \multicolumn{2}{|c|}{$\log g f$} \\
\hline & & & & & Ast. ${ }^{c}$ \\
\hline 4079.0719 & 0.92 & $80577_{1}$ & $105085_{2}$ & & 0.20 \\
\hline 4259.4126 & 0.78 & $82257_{3}$ & $105728_{4}$ & & 0.65 \\
\hline 4272.0440 & 0.97 & $82049_{2}$ & $105451_{3}$ & & -0.40 \\
\hline 4301.6974 & 0.97 & $82049_{2}$ & $105289_{3}$ & & 0.30 \\
\hline 4705.2854 & 0.96 & $84280_{1}$ & $105527_{2}$ & & 0.20 \\
\hline 4730.2672 & 0.95 & $88771_{2}$ & $109905_{2}$ & & 0.30 \\
\hline 4993.5338 & 0.98 & $89885_{1}$ & $109905_{2}$ & & 0.30 \\
\hline 5124.3561 & 0.98 & $88771_{2}$ & $108280_{3}$ & & 0.40 \\
\hline 5144.4921 & 0.89 & $69133_{0}$ & $88566_{1}$ & 0.01 & \\
\hline 5209.3246 & 0.84 & $69598_{1}$ & $88789_{2}$ & 0.38 & \\
\hline 5270.5120 & 0.95 & $69598_{1}$ & $88566_{1}$ & -0.31 & \\
\hline 5719.1384 & 0.96 & $69598_{1}$ & $87078_{0}$ & -0.41 & \\
\hline 6600.3388 & 0.95 & $69133_{0}$ & $84280_{1}$ & -0.40 & \\
\hline 6809.1955 & 0.97 & $69598_{1}$ & $84280_{1}$ & -0.05 & \\
\hline
\end{tabular}

${ }^{a}$ The normalized spectrum residual flux of the spectral line.

${ }^{b}$ Theoretical log $g f$ values from Palmeri et al. (2001).

${ }^{c}$ Astrophysically determined $\log g f$ values. The error in these values is dependant on the line intensity and the two stronger and the six weaker lines are estimated to have uncertainties of \pm 0.2 and \pm 0.4 dex, respectively.

of Kurucz (1993), while the Bi II hfs components from the present laboratory analysis were individually entered into the linelists.

The determination of the bismuth abundance in stellar spectra relies heavily on the existence and quality of the atomic oscillator strengths. During the past decades both theoretical (i.e. Gruzdev 1968; Bieron et al. 1991; Palmeri et al. 2001) and experimental oscillator strengths (Osherovich \& Tezikov 1978; Henderson et al. 1996) have been derived for selected Bi II lines, but there are notable discrepancies in the published data.

The determination of the bismuth abundance in the current work was made utilizing the theoretical $g f$ values of Palmeri et al. (2001). These values were calculated using the Hartree-Fock method with relativistic corrections and the multiconfiguration Dirac-Fock fully relativistic approach. The Palmeri et al. $\log g f$ values are presented for a number of UV and visible Bi II transitions, six of which can be seen in the optical spectrum of HR 7775 . It is difficult to assess the correctness of the theoretical $g f$ values, but the close agreement between the calculated values with the experimental $g f$ values of Henderson et al. (1996) for lines presented in both studies is an encouraging sign that the uncertainties in the calculations are small.

The bismuth abundance was determined from the two strongest Bi II lines, $\lambda 5144$ and $\lambda 5209$, with theoretical $g f$ values. From these lines the abundance was determined as $\log N_{\mathrm{Bi}}=5.80$ (on a scale where $\log N_{\mathrm{H}}=12$ ), indicating a 5 dex enhancement over the meteoritic value $\log N_{\mathrm{Bi}}=0.71$ (Grevesse \& Sauval 1998). This is consistent with the results derived from Wahlgren et al. (2001). The estimated fitting error of this abundance is \pm 0.1 dex. This error estimate is based on synthetic spectrum fits 
to the observed spectrum, and represent bismuth abundances that bracket the observed, noise-influenced, line profile. The determined abundance was then successfully utilized to obtain a fit to the four weaker Bi II features with theoretical $g f$ values. Thus, the abundance derived from the six transitions shows good agreement, which can be interpreted as a sign of consistency in the $g f$ value calculations of Palmeri et al.

The determination of the astrophysical $\log g f$ values in Table 6 was made utilizing the bismuth abundance determined from the Bi II features with theoretical $g f$ values. The $g f$ values were changed until a match was found between the synthetic and stellar spectra. Some of the Bi II features were very weak in the spectrum of HR 7775 (as indicated by their depths in Table 6), and the reality of their existence can only be substantiated by the broadness of the observed features. The weakness of most features for which astrophysical log $g f$ values have been determined complicates the spectrum fitting and the determined log $g f$ values are consequently quite uncertain, with an estimated uncertainty of \pm 0.4 dex. The astrophysical $g f$ values of the two strongest Bi II features in Table 6 , the $\lambda 4079$ and $\lambda 4259$ lines, have smaller estimated uncertainties of \pm 0.2 dex due to their greater line strength. The uncertainties in the $\log g f$ values represent limits in the line profile fitting as dictated by the noise level and any blending with unknown features.

The Bi II $\lambda 5209$ line is the strongest bismuth feature in the optical region of HR 7775 as measured by its equivalent width. As seen from the laboratory work this line has a hfs extending over approximately $1.2 \AA$, and most of the individual hfs components can be seen in the stellar spectrum. In Fig. 3a the laboratory spectrum of this line is shown and compared to the spectral features in HR 7775 (Fig. 3b).

It is interesting to note that most of the strongest laboratory $\mathrm{Bi}$ II features are present in the spectrum of HR 7775. In fact, only three transitions, at $\lambda 4204 \lambda 4227$ and $\lambda 4391 \AA$, with intensities larger than 20 in Table 1 are unobserved in HR 7775. Interestingly enough, these three features originate from noticably higher energy levels than the other strong optical Bi II lines. The strength of these features in the laboratory is a reflection of the overpopulation of certain high energy levels as a result of the experimental conditions pertaining to the charge transfer within the plasma (as discussed in Sect. 2).

From Table 6 it is seen that the Bi II $\lambda 4259$ and $\lambda 5209$ lines are the strongest of the optical lines as measured from their normalized flux, with the latter having a significantly broader hfs than the former. These lines are therefore most useful for detecting bismuth enhancements in the optical region of CP stars. The optical features of $\mathrm{Bi}$ II originate from energy levels high in the term system, and a substantial bismuth enhancement is needed in order to observe these lines. Much stronger features of Bi II can, however, be found in the UV region below $2000 \AA$ and these lines are therefore much better suited for the detection of a small to moderate abundance enhancement
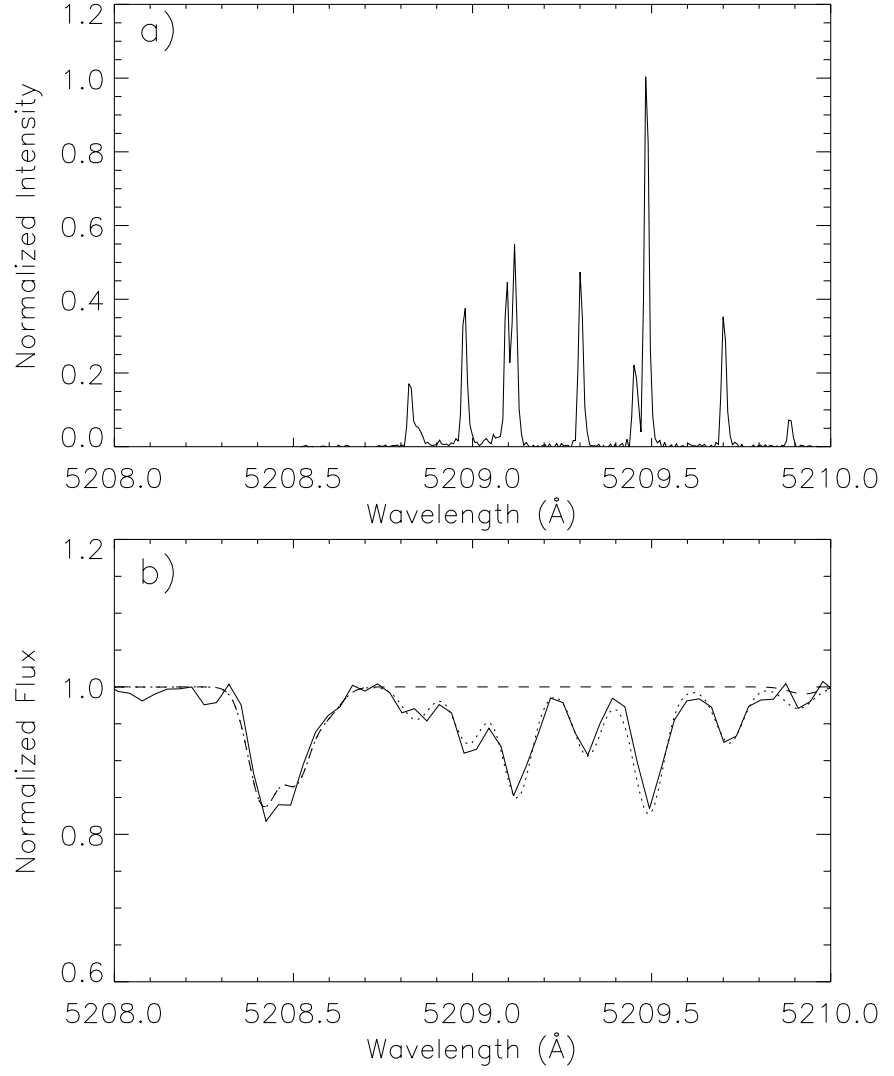

Fig. 3. The laboratory spectrum of the $\mathrm{Bi}$ II $\lambda 5209$ line a) is compared to the observation of HR $7775 \mathbf{b}$ ). For HR 7775 the observed spectrum (solid line) is compared with synthetic spectra for the solar abundance (dashed) and the best fit abundance (dotted) of bismuth.

of bismuth in the CP stars. Even though the laboratory work in the present paper only extends down to $2000 \AA$ the wavelengths and hfs of most lines below this limit can be established since the energy levels, $J$ values and hfs constants of almost all the energy levels below $130000 \mathrm{~cm}^{-1}$ are presented in our work.

Acknowledgements. The spectra used in this work were obtained with the Nordic Optical Telescope. The Nordic Optical Telescope is operated on the island of La Palma jointly by Denmark, Finland, Iceland, Norway, and Sweden, in the Spanish Observatorio del Roque de los Muchachos of the Instituto de Astroficica de Canarias.

\section{References}

Adelman, S. J. 1994, MNRAS, 266, 97

Arcimowicz, B., \& Dembczynski, J. 1979, Acta Phys. Pol. A, 56,661

Augustyniak, L., \& Werel, K. 1984, Phys. Scr., 30, 119

Bieron, J. R., Marcinek, R., \& Migdalek, J. 1991, J. Phys. B, 24,31

Bouazza, S., \& Bauche, J. 1988, Z. Phys. D, 10, 1

Cole, C. D. 1964, JOSA, 54, 859

Cowan, R. D. 1981, The Theory of Atomic Structure and Spectra (University of California Press, Berkeley). The Cowan code is available at different Web sites, e.g. 
http://plasma-gate.weizmann.ac.il, where a version adapted for PC by Ralchenko and Kramida can be found Cowley, C. R. 1987, Observatory, 107, 188

Crawford, M. F., \& McLay, A. B. 1934, Proc. R. Soc. London A, 143, 540

Edlén, B. 1966, Metrologia, 2, 71

Eisele, G., Koniordos, I., Müller, G., \& Winkler, R. 1968, Phys. Lett., 28B, 256

Fuhrmann, K. 1989, A\&AS, 77, 345

George, S., Munsee, J. H., \& Verges, J. 1985, JOSA B, 2, 1258

Grabowski, D., Drozdowski, R., Kwela, J., \& Heldt, J. 1996, Z. Phys. D, 38, 289

Grevesse, N., \& Sauval, A. J. 1998, SSRv, 85, 161

Gruzdev, P. F. 1968, Opt. Spectrosc., 25, 1

Guthrie, B. N. G. 1972, Ap\&SS, 15, 214

Guthrie, B. N. G. 1984, MNRAS, 206, 85

Henderson, M., Curtis, L. J., Ellis, D. G., Irving, R. E., \& Wahlgren, G. M. 1996, ApJ, 473, 565

Ilyin, I. 2000, Ph.D. Thesis, Univ. of Oulu

Jacobs, J. M., \& Dworetsky, M. M. 1982, Nature, 299, 535

Johansson, S. G., \& Litzén, U. 1978, J. Phys. B, 11, L703

Karlsson, H., \& Litzén, U. 2001, J. Phys. B, 34, 4475

Kurucz, R. L., \& Avrett, E. H. 1981, Smithsonian Astrophys. Obs. Spec. Rep., 391

Kurucz, R. L. 1993, Synthesis Programs and Line Data, (Kurucz CD-ROM No. 18)

Leckrone, D. S., Johansson, S. G., Wahlgren, G. M., Proffitt, C. R., \& Brage, T. 1998, in ASP Conf. Ser., 143, The Scientific Impact of the Goddard High Resolution
Spectrograph, ed. J. C. Brandt, T. B. Ake III, \& C. C. Petersen (San Fransisco: ASP), 135

Leckrone, D. S., Proffitt, C. R., Wahlgren, G. M., Johansson, S. G., \& Brage, T. 1999, AJ, 117, 1454

Moore, C. E. 1958, Atomic Energy Levels, 3 (NBS Circular, no. 467)(Washington, DC:US Govt Printing Office)

Murakawa, K., \& Suwa, S. 1947, Reports Inst. Sci. Tech., Tokyo Univ., 1, 121

Norlén, G. 1973, Phys. Scr., 8, 249

Osherovich, A. L., \& Tezikov, V. V. 1978, Opt. Spectrosc., 44, 128

Palmeri, P., Quinet, P., \& Biémont, E. 2001, Phys. Scr., 63, 468

Radziemski, L. J., Fischer, K. J., Steinhaus, D. W., et al. 1972, Comp. Phys. Commun., 3, 2

Smith, K. C., \& Dworetsky, M. M. 1993, A\&A, 274, 335

Stachowska, E. 1987, Ph.D. Thesis, University of Gdansk, Gdansk

Stachowska, E., Dembczynski, J., Arcimowicz, B., \& Kajoch, A. 1987, Z. Phys. D, 7, 177

Tuominen, I., Ilyin, I., \& Petrov, P. 1998, Astrophysics with the NOT, ed. H. Karttunen, \& V. Piirola (University of Turku: Turku), 47

Wahlgren, G. M., Brage, T., Gilliland, R. L., et al. 1994, ApJ, 435, L67

Wahlgren, G. M., Dolk, L., Kalus, G., et al. 2000, ApJ, 539, 908

Wahlgren, G. M., Brage, T., Brandt, J. C., et al. 2001, ApJ, 551,520 